\title{
On-line Automated Synthesis of Compact Normative Systems
}

\author{
JAVIER MORALES, Artificial Intelligence Research Institute (IIIA-CSIC), Spain \\ MAITE LÓPEZ-SÁNCHEZ, University of Barcelona, Barcelona, Spain \\ JUAN A. RODRIGUEZ-AGUILAR, Artificial Intelligence Research Institute (IIIA-CSIC), Spain \\ WAMBERTO VASCONCELOS, University of Aberdeen, United Kingdom \\ MICHAEL WOOLDRIDGE, University of Oxford, United Kingdom
}

\begin{abstract}
Most normative systems make use of explicit representations of norms (namely, obligations, prohibitions, and permissions) and associated mechanisms to support the self-regulation of open societies of selfinterested and autonomous agents. A key problem in research on normative systems is that of how to synthesise effective and efficient norms. Manually designing norms is time-consuming and error-prone. An alternative is to automatically synthesise norms. However, norm synthesis is a computationally complex problem. We present a novel on-line norm synthesis mechanism, designed to synthesise compact normative systems. It yields normative systems composed of concise (simple) norms that effectively coordinate a multiagent system without lapsing into over-regulation. Our mechanism is based on a central authority that monitors a multi-agent system, searching for undesired states. After detecting undesirable states, the central authority then synthesises norms aimed to avoid them in the future. We demonstrate the effectiveness of our approach through experimental results.
\end{abstract}

Categories and Subject Descriptors: I.2.11 [Artificial Intelligence]: Distributed Artificial Intelligence Multiagent Systems

General Terms: Algorithms

Additional Key Words and Phrases: Normative systems, norm synthesis

ACM Reference Format:

Javier Morales, Maite López-Sánchez, Juan A. Rodriguez Aguilar, Wamberto Vasconcelos, Michael Wooldridge. Automated Synthesis of Compact Normative Systems. ACM V, N, Article A (January YYYY), 33 pages.

DOI : http://dx.doi.org/10.1145/0000000.0000000

\section{INTRODUCTION}

A norm is an established pattern of behaviour which members of a society are expected to comply with [Bicchieri 2006]. Typically, norms impose restrictions on the behaviour of individuals. These behavioural restrictions play two important roles. On the one hand, they are of benefit to individuals because they simplify decision-making by ruling out various courses of action, thus reducing the decision space of alternatives

Author's addresses: Javier Morales, Artificial Intelligence Research Institute, Spanish Council of Scientific Research (IIIA-CSIC). Campus de la UAB, E-08193 Bellaterra, Catalonia (Spain); Maite López-Sánchez, MAiA Department, University of Barcelona. Gran Via, 58508007 Barcelona (Spain); Juan A. RodríguezAguilar, Artificial Intelligence Research Institute, Spanish Council of Scientific Research (IIIA-CSIC). Campus de la UAB, E-08193 Bellaterra, Catalonia (Spain); Wamberto Vasconcelos, Department of Computing Science, University of Aberdeen. Meston Building Meston Walk, Aberdeen, AB24 3UE, (United Kingdom); Michael Wooldridge, Department of Computer Science, University of Oxford. Wolfson Building, Parks Rd, Oxford OX1 3QD (United Kingdom).

Permission to make digital or hard copies of part or all of this work for personal or classroom use is granted without fee provided that copies are not made or distributed for profit or commercial advantage and that copies show this notice on the first page or initial screen of a display along with the full citation. Copyrights for components of this work owned by others than ACM must be honored. Abstracting with credit is permitted. To copy otherwise, to republish, to post on servers, to redistribute to lists, or to use any component of this work in other works requires prior specific permission and/or a fee. Permissions may be requested from Publications Dept., ACM, Inc., 2 Penn Plaza, Suite 701, New York, NY 10121-0701 USA, fax +1 (212) 869-0481, or permissions@acm.org.

(c) YYYY ACM 0000-0000/YYYY/01-ARTA $\$ 15.00$

DOI : http://dx.doi.org/10.1145/0000000.0000000 
that need to be considered. On the other hand, at the social level, they provide means whereby agents in societies can coordinate their activities, using only local decisionmaking [Binmore 2005].

Norms have been widely studied as a mechanism for coordinating multi-agent systems (MAS) [Shoham and Leyton-Brown 2009, Dignum 1999; Boella et al. 2006]. Coordination in this sense is usually understood as achieving some system-level goal, such as ensuring that the system avoids certain undesirable states. However, the problem of actually synthesising norms that effectively coordinate a multi-agent system is challenging. Since the seminal work of [Shoham and Tennenholtz 1995], the norm synthesis problem (namely, creating a set of norms which ensures that coordination is successful) has attracted considerable attention. Two approaches for norm synthesis have been considered in the literature: off-line and on-line.

Off-line approaches (e.g., [Shoham and Tennenholtz 1995; Fitoussi and Tennenholtz 1998]) are aimed at synthesising normative systems at design time. Unfortunately, the complexity of the off-line norm synthesis problem is high (NP-hard) [Shoham and Tennenholtz 1995]. These complexity issues have prompted research into the problem of managing the size of the system state space [Christelis and Rovatsos 2009]. Unfortunately, even if we ignore the problem of computational complexity, computing norms off-line is not appropriate if the state space of the system is not known in advance, or if it may change over time.

In contrast to off-line approaches, on-line approaches are aimed at synthesising norms at run-time rather than design time. The key conceptual advantage of on-line approaches compared to off-line approaches is that on-line approaches are not assumed to require complete knowledge of the system at design time.

Recently, norm emergence (or convention emergence) has become a popular technique for on-line norm synthesis (see, e.g., [Sen and Airiau 2007; Sen and Sen 2010; Griffiths and Luck 2010; Salazar et al. 2010; Villatoro et al. 2011; Yu et al. 2013]). Norm emergence does not require any global state representation or centralised control, and considers that agents collaboratively choose their own norms. Norm emergence therefore implies that agents are endowed with the computational capability to synthesise norms, and that they will choose to cooperate in the norm synthesis process. A norm is considered to have emerged when a significant number of agents in an agent society adhere to a common behaviour (that is, they choose the same actions), which is not dictated by a central authority. Therefore, a key issue in norm emergence is the design of emergence mechanisms that help agents agree on-line (converge) on some norm(s) [Kittock 1993; Walker and Wooldridge 1995]. Typically, state-of-the-art norm emergence mechanisms: (i) require an initial set of pre-designed, alternative norms; (ii) are sensitive to such initial conditions; and (iii) mainly converge to a unique norm instead of to a set of norms (with the exception of [Sen and Sen 2010; Salazar et al. 2010]).

A recent, alternative on-line approach is described in [Morales et al. 2011c]. There, norms are synthesised by observing agent interactions, without requiring their active participation in the synthesis process, unlike state-of-the-art norm emergence mechanisms. The approach in $\mid$ Morales et al. 2011c $\mid$ proved to be capable of synthesising a set of norms (i.e., a normative system), instead of a single norm, from scratch (namely, without requiring any initial, alternative norms). Moreover, it proved that the norms it synthesises are both effective and necessary to achieve MASs coordination. On the one hand, the effectiveness of a norm determines to what extent it is capable to avoid undesired MAS states. On the other hand, the necessity of a norm can be employed to determine whether it is really necessary to avoid undesired states, or those undesired states could be avoided without the existence of the norm. 
Typically, norms are synthesised with the aim of developing a stable set of norms that will prevent undesired states of the MAS (i.e., conflicts). However, assuming that agents are autonomous and they can choose whether to comply or not with norms, we cannot guarantee the prevention of undesired states. Therefore, here we assume that a set of norms is stable if: (i) norms avoid undesired states as long as agents comply with them, and (ii) the set of norms remains unchanged for an extended period of time. Norm stability is essential in order to provide agents with a common framework for their interactions. However, in addition to simply avoiding undesired states effectively, we might also want to consider compactness as a criteria for the on-line synthesis of norms. Compactness requires that the norms synthesised are as small as possible.

The work in [Morales et al. 2011c] (hereafter referred as BASE) considers compactness when synthesising norms. However, as we will show in this paper, it suffers from two major limitations. First, compactness is significantly jeopardised by the way synthesised norms are evaluated and generalised. On the one hand, the evaluation process is ill-defined because it unnecessarily aggregates the effectiveness and necessity of norms. On the other hand, general norms are synthesised with very little evidence, leading to the possibility of over-generalisation. Second, the approach of [Morales et al. 2011c] lacks stability: a set of norms is stable if they avoid undesired system states and remain unchanged for an extended period of time.

Notice that norm stability is essential in order to provide agents with a common framework for their interactions. As we will show below, the synthesis mechanism in [Morales et al. 2011c] is rather unstable, only being able to synthesise a normative system that avoids undesired states when the number of norm infringements in the system is very low. This means that this mechanism very frequently changes the rules of the game (the norms) to the agents in an agent society.

Against this background, the main contribution in this paper is a new, domainindependent norm synthesis mechanism called IRON (Intelligent Robust On-line Norm synthesis machine) to synthesise norms that characterise necessary conditions for coordination, while avoiding over-regulation. Likewise BASE, IRON (available in the online appendix) assumes that (1) norms do not incorporate sanctions, and hence they can be regarded as soft-constraints imposed to the agents; and (2) agents employ a stochastic norm infringement model, although rational norm infringements would guide towards useful normative systems in a more informed way. In this realm, we show that IRON significantly outperforms the approach described in [Morales et al. 2011c] in terms of:

- Stability. Unlike the approach in [Morales et al. 2011c], IRON is highly stable, capable of synthesising enduring normative systems that avoid undesired states despite a high number of infringements in the agent society. We explore the limits of IRON to empirically show that its synthesis mechanism manages to synthesise norms even when the probability of agents violating norms is high, namely when half of the decisions of each agent may result in norm infringements.

- Compactness. IRON manages to converge to normative systems that are between $30 \%$ and 70\% more compact (have fewer norms) than those synthesised by the approach of [Morales et al. 2011c].

These advantages stem from the core components of IRON's abstract architecture, namely: (i) an evaluation method that evaluates norms in terms of different synthesis criteria, which allows IRON to be stable; (ii) a generalisation operator that allows IRON to synthesise compact normative systems, only generalising norms when there is enough evidence; and (iii) a specialisation operator that makes it possible to undo under-performing generalisations. 
Notice that although IRON was originally introduced in [Morales et al. 2013], we provide several extensions in this paper. First, here we provide a comprehensive description of IRON's norm generalisation mechanism, which is a core process within its synthesis process. Second, we introduce metrics for compactness of normative systems, and provide a thorough empirical evaluation of IRON's compactness. Third, we provide a detailed comparison of IRON versus the approach in [Morales et al. 2013] in terms of stability and compactness.

The proposed approach can be then applied to a wide range of domains that can be regulated by means of norms. As an example, in a traffic scenario IRON can be employed to synthesise traffic rules that prevent cars from colliding. Another example is the case of on-line communities, where individuals interact by uploading and sharing opinions about different contents. There, users can also complain about those contents they find to be inappropriate. In [Morales et al. 2014], IRON has been proven to be capable of synthesising norms that prevent the users of an on-line community from uploading inappropriate contents.

The remainder of this paper is organised as follows. Section 2 introduces the basic framework within which we work, and defines the norm synthesis problem that we tackle in this paper. Section 3 summarises the approach of [Morales et al. 2011c] in order to facilitate comparison with IRON, which is described in Section 4 Section 5 presents our empirical evaluation of IRON. Finally, Section 6 draws some conclusions and discusses on the applicability and limitations of our approach. Finally, Section 7 describes some possible avenues for future research.

\section{BASIC DEFINITIONS AND PROBLEM STATEMENT}

In this section we provide some basic definitions and use these to formally state the problem of norm synthesis.

\subsection{Basic definitions}

We consider a system composed of a finite set of agents $A g=\left\{a g_{1}, \ldots, a g_{n}\right\}$ with a shared finite set of actions $A c=\left\{a c_{1}, \ldots, a c_{m}\right\}$ that these agents can perform. Let $S$ be the set of all possible states of the system, and let $C \subseteq S$ be a set of undesired (conflicting) states. We do not require any specific semantics for the notions of state and undesired state: the interpretation will depend on the particular domain of interest. As an example, consider a traffic scenario in which agents correspond to computercontrolled cars. In this case, the set of undesired states would correspond to those states containing, e.g., collisions.

We will use a language $\mathcal{L}$ to describe the states of a MAS. This language, to be more formally defined later, is a logical language containing the standard classical connectives, and a notion of consequence defined for it via a relation $\models$. Given a state $s \in S$, we let $\nu(s)$ denote an expression in $\mathcal{L}$ that describes the state. For instance, if $\mathcal{L}$ is a predicate logic language with grounded terms and $s$ stands for a state of a traffic junction, $\nu(s)$ would be composed of the predicates describing the position of each car in it.

We assume that each agent has its own local view of the state of the system that it is part of. For instance, a car located at a road junction will have its own local perception of the system, which corresponds to the perception of cars in its vicinity (i.e., the junction) without including those other cars further away in the road. Thus, an agent context is an agent's internal representation of a system state (i.e., its beliefs). Agents express their contexts in terms of an agent language $\mathcal{L}_{A g}$, which we will detail at a later point. However, we often find it convenient to assume that the language is that of a predicate logic with grounded terms. We denote the consequence relation for this 
logic as $=$. Henceforth, given a state $s \in S$ and an agent $a g \in A g$ that is part of $s$, we will refer to its context by means of function $c: A g \times S \rightarrow \mathcal{L}_{A g}$.

We now introduce our notion of norm, which establishes obligations, permissions and/or prohibitions [Meyer and Wieringa 1993] to an individual agent whenever some pre-conditions are fulfilled. Since in this work we focus on synthesising norms that can be easily interpreted and fulfilled by agents, we employ the agent language to express norms. Therefore, norm pre-conditions are expressed as formulae of $\mathcal{L}_{A g}$, and hence in terms of an agent's point of view.

Definition 1 (NORM). A norm is a pair $\langle\varphi, \theta(a c)\rangle$ where $\varphi \in \mathcal{L}_{A g}$ stands for the precondition of the norm, ac $\in A c$ is an action, and $\theta \in\{$ obl,perm, prh $\}$ is a deontic operator: obl indicates an obligation, perm indicates a permission, and prh indicates a prohibition.

An agent $a g \in A g$ evaluates whether a norm $n=\langle\varphi, \theta(a c)\rangle$ applies in a state $s$ as follows. First, we say that the context of $a g$ in $s, c(a g, s)$, satisfies the pre-condition of norm $n$ if, and only if, $c(a g, s) \models \varphi$. In this case, norm $n$ applies to agent $a g$ and the deontic expression $\theta(a c)$ will hold for it. More concretely, we assume the precondition $\varphi$ is a set of first-order predicates $p\left(\tau_{1}, \ldots, \tau_{n}\right)$, with $p$ being a predicate symbol and $\tau_{1}, \ldots, \tau_{n}$ being terms of $\mathcal{L}_{A g}$ (the set of predicates represents their conjunction); $\theta(a c)$ is an atomic deontic formula. We represent a normative system $\Omega$ as a set of norms.

Notice that a normative system in our model essentially consists of a set of soft constraints on the behaviour of agents. It could be argued that this approach is more specific than is strictly necessary: an alternative approach would be for a norm designer simply to specify the undesirable system states (conflicts) that agents should avoid. The advantage of such an alternative approach is that it provides agents with more flexibility than IRON: it leaves an agent free to choose how to respect the norm. There are at least two difficulties with such an alternative approach, however. First, it presents agents with the problem of determining for themselves how to act in such a way as to respect the norm; that is, each agent should then solve a potentially complex norm compliance problem. Secondly, such an approach may cause problems if there are multiple possible norms that satisfy the designers intent, each involving multiple agents. In this case, communication and cooperation may be required in order to ensure that agents correctly coordinate on the selection of just one of the available set of norms. At the very least this will impose an additional communication overhead on agents, and at worst, it may be impossible in time-constrained settings.

We now introduce a running example to be used throughout the remainder of the paper. We consider a traffic junction scenario composed of two orthogonal roads. Agents are autonomous cars that enter the scenario and cross the junction to reach their destinations. A car perceives the scenario by means of its local context, which is expressed by means of three unary predicate symbols $\{$ left, front, right $\}$ of $\mathcal{L}_{A g}$, which are used to represent what occupies the three road positions the car perceives. Each predicate has a single term from $\{c a r$, bike, private, ambulance, police, fire-brigade, emergency, nil $\}$ of $\mathcal{L}_{A g}$, representing different vehicle types, and the symbol "nil" standing for no vehicle. The actions available to agents are $A c=\{G o, S t o p\}$. In particular, the action Go means that the car moves forward in its target direction (in particular it may include turns), and Stop means that the agent remains stopped in its position.

Using this traffic scenario, we can represent a norm that establishes a prohibition to go for an agent that observes a car to its left (hence giving way to it) as:

$$
n_{0}:\langle\{\operatorname{left}(\operatorname{car}), \text { front }(\text { nil }), \operatorname{right}(\text { nil })\}, \operatorname{prh}(\text { Go })\rangle
$$


Where the precondition has a predicate left(car) that is true if there is a car to the left of the agent evaluating the norm (the reference car), front (nil) and right(nil) are predicates that are true when, respectively, the front and right positions of the reference car are empty. So, if agent ag's context is $\{$ left(car), front $(n i l)$, right $(n i l)\}$, then, $c(a g) \models \varphi$ holds and $\operatorname{prh}(G o)$ applies to the car.

Norm $n_{1}$ below prohibits a car from going (hence giving way) if there is an ambulance to its left, a car to its front and nothing to its right; it works in conjunction with two additional norms $n_{2}$ and $n_{3}$ to regulate priority of emergency vehicles:

$$
\begin{aligned}
& n_{1}:\langle\{\text { left }(\text { ambulance }), \text { front }(\text { car }), \operatorname{right}(\text { nil })\}, \operatorname{prh}(\text { Go })\rangle \\
& n_{2}:\langle\{\text { left }(\text { police }), \quad \text { front }(\text { car }), \operatorname{right}(\text { nil })\}, \operatorname{prh}(G o)\rangle \\
& n_{3}:\langle\{\text { left }(\text { fire-brigade }), \text { front }(\text { car }), \operatorname{right}(\text { nil })\}, \operatorname{prh}(G o)\rangle
\end{aligned}
$$

Let us suppose that $\left\{n_{1}, n_{2}, n_{3}\right\}$ comprise a normative system $\Omega$. We observe that we can generalise these norms into a single norm that regulates when to give way to emergency vehicles coming from the left:

$$
n_{5}:\langle\{\text { left(emergency), front(car), right(nil) }\}, \operatorname{prh}(\text { Go })\rangle
$$

As we explain below, this generalisation is possible because $n_{5}$ caters for the same situations (pre-conditions) of $\left\{n_{1}, n_{2}, n_{3}\right\}$, and establishes the same norm. Notice thus that whenever norms $n_{1}, n_{2}$ or $n_{3}$ are applicable, their parent norm $n_{5}$ will be applicable as well. Let us now consider a norm similar to $n_{3}$, which prohibits a car from going if there is a fire-brigade vehicle to its left, nothing to its right, and there is a bike in front:

$$
n_{4}:\langle\{l e f t(\text { fire-brigade }), \text { front }(\text { bike }), \operatorname{right}(\text { nil })\}, \operatorname{prh}(\text { Go })\rangle
$$

Similarly to the generalisation of $n_{1}, n_{2}$, and $n_{3}$ into $n_{5}$, we may generalise $n_{3}$ and $n_{4}$ as a single norm establishing that agents should not go (and thus yield to a firebrigade) when there is a private vehicle in front (either a car or a bike):

$$
\left.n_{6}:\langle\{\text { left(fire-brigade), front(private), right }(\text { nil })\}, \operatorname{prh}(\text { Go }))\right\rangle
$$

Next, we define how to establish such relationship between norms. We use a subsumption relationship ( $\sqsubseteq$ ) between the terms in $\mathcal{L}_{A g}$. Thus, if $\tau, \tau^{\prime} \in \mathcal{L}_{A g}$ and $\tau^{\prime} \sqsubseteq \tau$, we say that $\tau$ is more general than $\tau^{\prime}$. In our example, police $\sqsubseteq$ emergency, as a police car "is a" specific kind of emergency vehicle.

DEFINITION 2 (NORM GENERALISATION). We say that norm $n=\langle\varphi, \theta(a c)\rangle$ is more general than norm $n^{\prime}=\left\langle\varphi^{\prime}, \theta(a c)\right\rangle$, denoted as $n^{\prime} \subseteq n$, iff $|\varphi|=\left|\varphi^{\prime}\right|$, and for each predicate $p\left(\tau_{0}^{\prime}, \ldots, \tau_{n}^{\prime}\right) \in \varphi^{\prime}$, there is a predicate $p\left(\tau_{0}, \ldots, \tau_{n}\right) \in \varphi$ such that $\tau_{i}^{\prime} \sqsubseteq \tau_{i}, 0 \leq i \leq n$.

In general, if $n_{j}$ is generalised by $n_{i}$, then we also say that $n_{i}$ is specialised by $n_{j}$. If there exists at least some $n_{k} \in \mathcal{N}$ such that $n_{j} \subseteq n_{k} \subseteq n_{i}$ we say that $n_{i}$ is an ancestor of $n_{j}$, otherwise $n_{i}$ is a father of $n_{j}$. If $n_{j}$ is not generalised by $n_{i}$, we denote it by $n_{j} \nsubseteq n_{i}$.

\subsection{Research problem}

We evaluate norms and normative systems in terms of their effectiveness and necessity in achieving coordination. On the one hand, our system IRON measures the cumulative effectiveness of a norm from the outcomes of its fulfilments: the higher the ratio of successful fulfilments (fulfilments that did not lead to an undesired MAS state), the more 
effective the norm. On the other hand, it measures the necessity of a norm according to the following principle: the higher the ratio of harmful infringements (infringements leading to undesired states), the more necessary the norm.

In this paper, we focus on a particular type of MAS, namely normative multi-agent system (NMAS). A NMAS is one whose agents have their actions regulated by some normative system (set of norms) they are aware of. Moreover, the system itself can assess whether and to whom norms in the normative system apply. For the sake of clarity, although NMAS is a concept used in the literature [Singh et al. 2013], the specific notion of NMAS we use is as follows:

Definition 3 (NoRMATIVE MAS). A Normative Multi-agent System (NMAS) is a tuple $\left\langle A g, A c, \Omega, \mathcal{L}_{A g}, S_{0}, S\right\rangle$, where: (i) Ag is a set of agents; (ii) Ac is a set of actions; (iii) $\Omega$ is a normative system, whose norms are expressed in the agent language $\mathcal{L}_{A g}$; (iv) $S_{0}$ is the initial MAS state and; (v) $S$ is a set of states.

Given a NMAS, our aim is to generate a normative system that avoids undesired states as long as agents comply with them, while avoiding over-regulation. With this aim, given a normative system we must be able to measure: (i) the effectiveness of its norms in preventing undesired states when agents comply with them; and (ii) whether its norms are necessary to prevent undesired states (that is, if agents infringing norms lead to undesired states or not). Furthermore, since the state of a NMAS changes as agents interact, our goal is to find a stable normative system, namely a set of norms that remains unchanged and whose norms are sufficiently effective and necessary for a given period of time. With this aim, we make use of functions $\mu_{\text {eff }}$ and $\mu_{n e c}$, which are detailed in Section 4.3, that measure the effectiveness and necessity of a norm, respectively. We use these functions in tandem with: (i) a time period $\mathbb{T}$ in which the norms of the normative system are required to be sufficiently effective and necessary to consider the normative system as stable; and (ii) threshold values $\alpha_{\text {eff }}^{\text {spec }}$ and $\alpha_{n e c}^{\text {spec }}$, where $\alpha_{\text {eff }}^{\text {spec }}, \alpha_{\text {nec }}^{\text {spec }} \in[0,1]$. These thresholds set satisfaction degrees for effectiveness and necessity. Thus, any norm which effectiveness or necessity is under respective thresholds, will be removed from the normative system. Finally, we define the problem that we address in this paper:

Definition 4 (Norm Synthesis Problem). Let $\left\langle A g, A c, \Omega, \mathcal{L}_{A g}, S\right\rangle$, be a NMAS, $C \subseteq S$ a set of undesired states, $\mu_{\text {eff }}, \mu_{\text {nec }}$ functions to assess the effectiveness and necessity of a norm, $\alpha_{\text {eff }}, \alpha_{\text {nec }}$ satisfaction degrees, $\psi$ a function that returns the normative system at each given time $t, t_{0}$ the initial time step, and $T=\left[t_{\text {begin }}, \ldots, t_{\text {end }}\right]$ a time interval. The norm synthesis problem (NSP) is that of finding a normative system $\bar{\Omega}$ such that for some $t_{\text {begin }} \geq t_{0}$, and for all $t$ in $T$, the following conditions hold: (1) for each $n \in \psi(t), \mu_{e f f}(n, t, C)>\alpha_{\text {eff }}^{\text {spec }}$ and $\mu_{n e c}(n, t, C)>\alpha_{n e c}^{\text {spec }}$; and $(2) \psi(t)=\bar{\Omega}$.

Notice that solving this problem amounts to finding a stable normative system for a given period of time. Of course, it may be that several normative systems might be sufficiently effective and necessary during a period of time. To further distinguish between them, we use a measure of compactness: we prefer smaller normative systems over larger ones.

DEFINITION 5 (COMPACTNESS). The compactness of a normative system $\Omega$ is the total number of predicates in its norms, namely $\sum_{n \in \Omega}|n|$, where $|n|$ stands for the number of predicates in a norm. 


\section{BACKGROUND}

In this section we survey BASE, the norm synthesis approach described in [Morales et al. 2011c], as our work builds on this framework. BASE is an iterative approach that continuously monitors a system, searching for undesired states. Whenever an undesired state is detected, a norm generation process is initiated, which results in the generation of a new norm aimed at avoiding that undesired state in the future. This generation process is based on an unsupervised approach of Case-Based Reasoning (CBR). Classical CBR [Riesbeck and Schank 1989;, Aamodt and Plaza 1994] is a supervised machine learning technique that solves new problems (i.e., cases) by obtaining similar ones from a knowledge base (i.e., a case base) and adapting their solutions under the supervision of an expert. The unsupervised CBR in [Morales et al. 2011c] starts with an initially empty case base, and does not require an expert to evaluate generated solutions. Instead, cases (and their solutions) are elicited at runtime, and evaluated in an unsupervised manner. Whenever BASE detects an undesired MAS state, it generates (and adds to the case base) a new case that describes the undesired state (at time $t$ ), and the previous state (at time $t-1$ ) that led to that undesired state 11 . Then, it generates a new solution that is aimed to avoid the transition to that undesired state in the future. To generate solutions, it randomly chooses one of the agents involved in the transition (for instance, in a traffic scenario, one of the agents that have collided). Then, it prohibits the action that the agent performed in the transition from the previous to the undesired state (that is, from time $t-1$ to $t$ ). The generated solution is finally translated into a norm that agents can interpret and comply with. Generated norms (and their corresponding solutions) are evaluated in runtime based on their outcomes in the MAS.

Next, a norm evaluation process is carried out, which computes the performance of of those norms that have been fulfilled, as well as those that have been infringed, during the current step. Finally, a norm refinement process is carried out, which discards norms that under-perform during a given period of time $T_{w}$.

Finally, as a result of norm generation, evaluation and refinement, BASE outputs a normative system $\Omega$. This normative system, which contains the currently prevailing norms, is then broadcast to the agents so that they become aware of it.

In order to provide the proper background to our approach, we present Algorithm 1 from [Morales et al. 2011c]. It describes the BASE strategy for synthesising normative systems. In the norm generation phase, for each detected undesired MAS state (i.e., conflict), the function generateNorm (line 2) creates a new norm aimed at avoiding that particular state, and adds the new norm to the normative system $(\Omega)$. Next, the norm evaluation process evaluates applicable norms, that is, those norms that have been fulfilled $(F)$ and infringed $(I)$ during the current step $t$ (lines 4-5), in terms of their effectiveness and necessity to achieve MASs coordination. On the one hand, it measures the cumulative effectiveness of a norm from the outcomes of its fulfilments. Specifically, whenever agents fulfil a norm and it leads to a non-undesired state (i.e., a non-conflicting state), then the norm is considered as effective to avoid undesired states. By contrast, if agents comply with the norm and it leads to an undesired state, then the norm is considered as ineffective. Thus, the higher the ratio of successful fulfilments (fulfilments that did not lead to undesired MAS states), the more effective the norm. On the other hand, it measures the necessity of a norm based on the outcomes of its infringements. Whenever agents infringe a norm and it leads to an undesired state, then the norm is considered as necessary. If agents infringe the norm and it does not lead to an undesired state, then it is considered as unnecessary. Thus, the higher the

\footnotetext{
${ }^{1}$ This implies that BASE must continuously monitor the MAS by means of a window of two time steps (that
} is, the current MAS state at time $t$ and the previous MAS state at time $t-1$ ). 


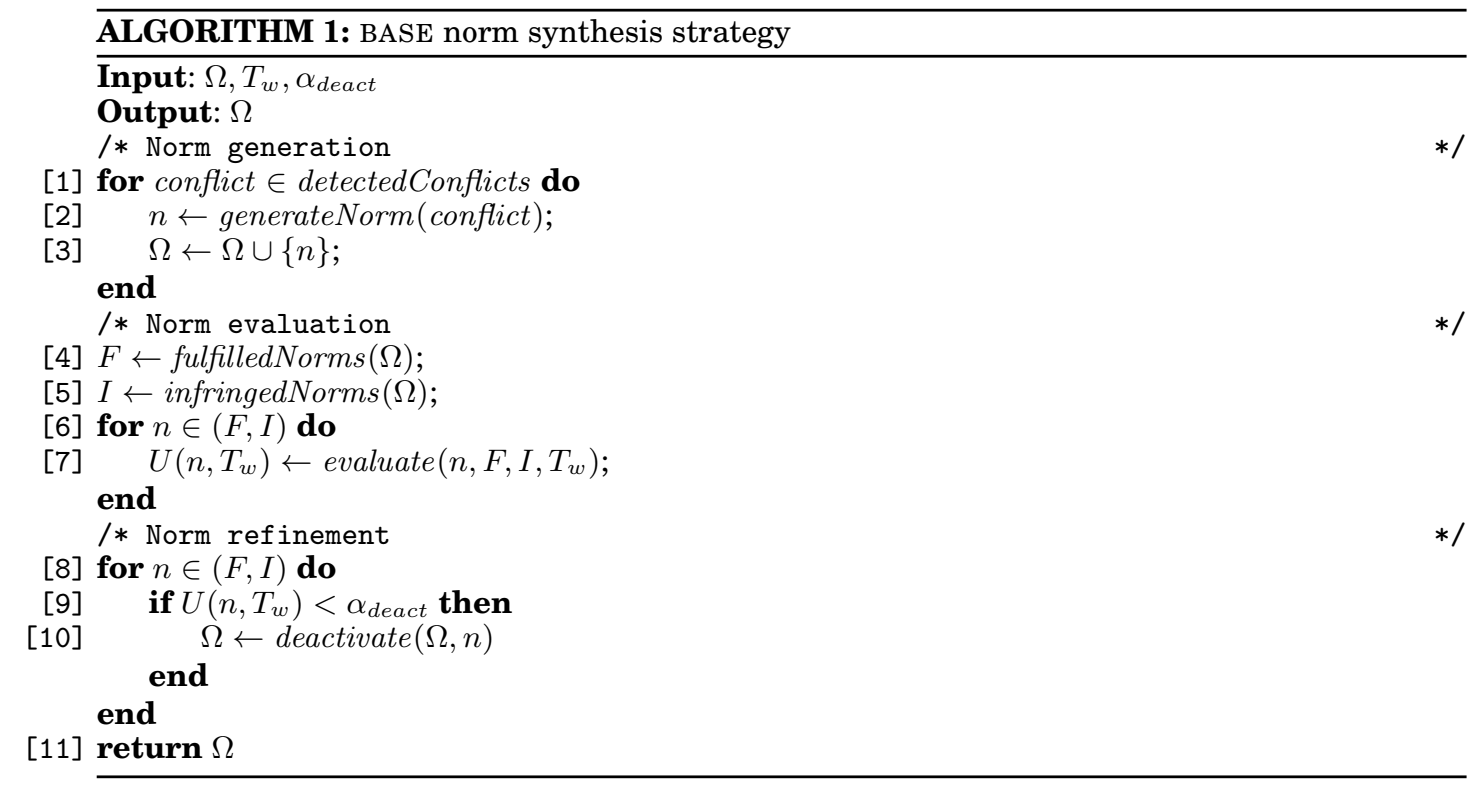

ratio of harmful infringements (infringements leading to undesired MAS states), the more necessary the norm. The function evaluate (line 7) evaluates applicable norms by combining their effectiveness and necessity into a single utility measure $u$. Finally, the overall utility $U$ of a norm is computed as the degree of positive evaluations (i.e., $u>0$ ) over its total evaluations during a period of time $T_{w}$ :

$$
U\left(n, T_{w}\right)=\frac{\bar{u}_{+}\left(n, T_{w}\right)}{\bar{u}\left(n, T_{w}\right)}
$$

where $\bar{u}_{+}\left(n, T_{w}\right)$ stands for the average of the positive evaluations of $n$ during the period of time $T_{w}$ and $\bar{u}\left(n, T_{w}\right)$ stands for the average of all evaluations of $n$ during period $T_{w}$. Finally, BASE carries out a norm refinement process, which deactivates those norms that under-performed during period $T$. Whenever the utility $U\left(n, T_{w}\right)$ of a norm is under a certain threshold $\alpha_{\text {deact }}$, it is deactivated (line 10).

This approach can be seen to suffer from the following drawbacks:

- Generalisation on the basis of scarce evidence - Let us consider a traffic scenario and the following situation: a car perceives another car to its left, nothing in front and nothing to its right. Let us suppose that we want to force a car to stop whenever it perceives this situation. An appropriate norm to generate would be: "stop when there is a car to your left, nothing in front and nothing to your right". However, we observe that this norm synthesis instead generates a general norm such as "stop when there is a car to your left," disregarding the front and right positions. This norm should only be generated when there is evidence of all the situations it may represent. However, BASE generates this general norm on the basis of a single piece of evidence.

- Ill-defined evaluation - The norm evaluation process evaluates norms by aggregating their effectiveness and necessity into one unique value that represents their overall utility. This causes a coupling effect between effectiveness and necessity, and makes it impossible to evaluate whether norms are necessary independently of their effectiveness. For instance, a norm that is highly unnecessary but also highly effective 
may be evaluated as performing well, since its resulting utility balances the differences between its effectiveness and necessity.

- Lack of specialisation - This problem arises when norms that do not perform well are simply removed from the normative system, without taking into account the fact that under-performing general norms may be refined to eliminate just those cases that decrease their utility.

Despite the above-mentioned drawbacks, one of the advantages of BASE is its low complexity, as shown by the following lemma.

LEMMA 3.1. The complexity of the norm synthesis performed by the BASE algorithm employing a case base $C$ containing $\eta_{C}$ norms when detecting $\kappa$ conflicts is $O\left(\kappa \cdot \eta_{C}+4\right.$. $|A g| \cdot|\Omega|)$.

PROOF. On the one hand, the cost of generating norms for all detected conflicts is $O\left(\kappa \cdot \eta_{C}\right)$, since it may require to search through the whole case-based reasoning base per conflict. Next, the cost of building the sets $F$ and $I$ of fulfilled and infringed norms is $O(2 \cdot|A g| \cdot|\Omega|)$. This is because each norm in the normative system may be either fulfilled or infringed by each agent in the system. Since the total size of the sets $F$ and $I$ together is $|A g| \cdot|\Omega|$, the cost of evaluating norms is $O(|A g| \cdot|\Omega|)$. Therefore, altogether the norm evaluation process is $O(3 \cdot|A g| \cdot|\Omega|)$. Finally, the cost of the norm refinement process is $O(|A g| \cdot|\Omega|)$ since, again, it requires a search over a list whose maximum size is $|A g| \cdot|\Omega|$. Putting all together, the resulting worst-case complexity is $O\left(\kappa \cdot \eta_{C}+4 \cdot|A g| \cdot|\Omega|\right)$.

\section{IRON: A NORM SYNTHESIS MECHANISM}

In this section we introduce the Intelligent Robust On-line Norm synthesis mechanism (IRON), a norm synthesis approach aimed at solving the norm synthesis problem formalised by Definition 4. IRON is intended to synthesise effective compact normative systems (that is, normative systems that avoid undesired MAS states).

While the basic operation of IRON follows the on-line approach of the BASE algorithm described in Section 3, IRON was designed to overcome the drawbacks of BASE. Specifically, given a NMAS, IRON operates by continuously iterating the following steps: (1) it monitors the operation of the system; (2) it decides upon the addition of brand new norms to the current normative system; (3) it evaluates whether the effectiveness and necessity of the normative system are within expected thresholds; (4) if required, it refines the normative system; and (5) it makes the normative system available to the agents. Notice therefore that IRON continuously searches for a normative system online, while agents in the system are operating.

IRON is based on five components, namely, (i) a grammar for the synthesis of new norms; (ii) the normative network (a data structure to represent normative systems and explored norms); (iii) a method for the evaluation of norms and normative systems; (iv) a set of operators that make it possible to transform one normative system into another; and (v) a strategy that specifies when to use such operators. We describe below each component in detail, and explain IRON's architecture and computational model.

\subsection{Grammar for norm synthesis}

Our approach employs a grammar $\mathcal{G}$ to synthesise candidate norms of the form $\langle\varphi, \theta(a c)\rangle$ (cf. Def 1). We have adapted our grammar from [García-Camino et al. 2009], using as building blocks atomic formulae of the form $p^{n}\left(\tau_{1}, \ldots, \tau_{n}\right), p$ being an n-ary predicate symbol and $\tau_{1}, \ldots, \tau_{n}$ terms of $\mathcal{L}_{A g}$. 


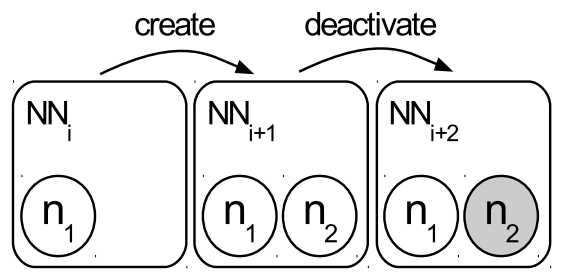

Fig. 1. Evolution of a normative network (and its normative system) along time: $\Omega_{i}=\left\{n_{1}\right\}, \Omega_{i+1}=$ $\left\{n_{1}, n_{2}\right\}, \Omega_{i+2}=\left\{n_{1}\right\}$

$$
\begin{array}{ll}
\text { Norm } & ::=\langle\{\text { LHS }\}, \text { RHS }\rangle \\
\text { LHS } & ::=\text { LHS, LHS } \mid \rho \\
\text { RHS } & ::=\theta(A c) \\
\theta & ::=o b l \mid \text { perm } \mid \text { prh } \\
A c & ::=a c_{1}\left|a c_{2}\right| \ldots \mid a c_{n} \\
\rho & ::=p^{n}\left(\tau_{1}, \ldots, \tau_{n}\right) \\
\tau & ::=c_{1}|\ldots| c_{n}\left|x_{1}\right| \ldots \mid x_{m}
\end{array}
$$

where $a_{i}$ and $x_{j}$ are constants and variables, respectively. We represent as $\mathbf{N}$ the set of all norms which comply with the grammar above.

If we consider further our traffic scenario, the grammar used to synthesise the norm examples given in Section 2 is as follows:

$$
\begin{array}{ll}
\text { Ac } & ::=\text { Go } \mid \text { Stop } \\
\rho & ::=\operatorname{left}(\tau) \mid \text { front }(\tau) \mid \operatorname{right}(\tau) \\
\tau & ::=\text { car } \mid \text { bike } \mid \text { private } \mid \text { ambulance } \mid \text { police } \mid \text { fire-brigade | emergency | nil }
\end{array}
$$

Terms $\tau$ in this grammar have the following subsumption relationships: car $\sqsubseteq$ private, bike $\sqsubseteq$ private, ambulance $\sqsubseteq$ emergency, police $\sqsubseteq$ emergency, fire-brigade $\sqsubseteq$ emergency.

\subsection{A representation of normative systems}

Since IRON will continuously synthesise norms in search for a normative system, it must be able to differentiate between the norms that are currently part of the normative system and those that are not (i.e., they have been explored but they are not currently active). For this purpose, IRON employs a graph-based data structure to represent normative systems, which we call a normative network. A normative network is a graph whose nodes stand for norms and whose edges stand for relationships (more specifically, in our work we concentrate on generalisation relationships) between norms. Norms in a network may be either active or inactive. We assume that a normative network represents a normative system as its active norms.

Figure 1 illustrates the evolution of a normative network (and its corresponding normative system) over time points $t_{i}, t_{i+1}, t_{i+2}$. At time $t_{i}$ the normative network $N N_{i}$ has a single active norm $n_{1}$ (represented as a white circle) and $\Omega_{i}=\left\{n_{1}\right\}$. At time $t_{i+1}$ a new norm, $n_{2}$, is added to $N N_{i}$, yielding $N N_{i+1}$ and $\Omega_{i+1}=\left\{n_{1}, n_{2}\right\}$. Finally, at time $t_{i+2}$ norm $n_{2}$ is deactivated (represented as a gray circle) giving rise to $N N_{i+2}=\left\{n_{1}, n_{2}\right\}$ and $\Omega_{i+2}=\left\{n_{1}\right\}$. Figure 1 also illustrates the way IRON performs the norm synthesis process. In general, the process will consist of continuously iterating over (applying changes to) the normative network according until it finds a normative system that solves the norm synthesis problem (NSP).

We now offer a formal definition of the normative network employed by IRON: 
Definition 6 (NoRmative Network). A Normative Network (NN) is a tuple $\left\langle\mathcal{N}, R_{G}, \Delta, \delta\right\rangle$ where: (i) $\mathcal{N} \subseteq \mathbf{N}$ is a subset of our language of norms; (ii) $R_{G} \subseteq \mathcal{N} \times \mathcal{N}$ is a generalisation relationship between norms; (iii) $\Delta=\{$ active, inactive $\}$ is the set of possible states of a norm; (iv) $\delta: \mathcal{N} \rightarrow \Delta$ is a function that returns the state of a norm $n \in \mathcal{N}$.

Since IRON considers that the current normative system is composed of the norms that are currently active in the normative network, we define $\Omega=\{n \mid n \in \mathcal{N}, \delta(n)=$ active\}.

\subsection{Evaluating norms and normative systems}

We detail how we evaluate norms and normative systems in terms of their effectiveness and necessity to achieve MASs coordination. IRON evaluates these two measures individually, along the lines of BASE (described in Section 35). On the one hand, it measures the cumulative effectiveness of a norm from the outcomes of its fulfilments. The higher the ratio of successful fulfilments (fulfilments that did not lead to undesired states), the more effective the norm. On the other hand, it measures the necessity of a norm according from the outcomes of its infringements. The higher the ratio of harmful infringements (infringements leading to undesired states), the more necessary the norm is. We illustrate norm evaluation with the following example. In a traffic scenario, consider a norm like "give way to right". This is an effective norm, since agents will not collide with the car to their right as long as they comply with this norm. Thus, whenever agents fulfil this norm it will lead to states that are not undesired (i.e., states without car collisions), and that is why we call them successful fulfilments. Furthermore, this norm is also necessary to prevent cars from colliding with the cars to their right. Therefore, whenever agents infringe the norm it will lead to undesired states (i.e., states where cars have collided). This is what we call a harmful infringement.

We compute the effectiveness $\mu_{\text {eff }}(n, t, C)$ of norm $n$ up to time $t$ as:

$$
\mu_{\text {eff }}(n, t, C)=(1-\alpha) \times \mu_{\text {eff }}(n, t-1, C)+\alpha \times r_{\text {eff }}(n, t, C)
$$

where $\mu_{\text {eff }}(n, t-1, C)$ is the effectiveness of $n$ up to time $t-1$ and $0 \leq \alpha \leq 1$ is a learning rate; and $r_{\text {eff }}(n, t, C)$ is a reward value based on the successful fulfilments of norm $n$ at time $t$. In particular, at an initial time $t_{0}$, the effectiveness of a norm, namely $\mu_{e f f}\left(n, t_{0}, C\right)$ is set with an initial constant value $k \in[0,1]$. The reward of a fulfilled norm at time $t$ is computed as:

$$
r_{e f f}(n, t, C)=\frac{w_{S F} \times m_{S F}(n, t, C)}{w_{S F} \times m_{S F}(n, t, C)+w_{H F} \times m_{H F}(n, t, C)}
$$

where $m_{S F}(n, t, C)$ is the number of successful fulfilments of norm $n$ at time $t$, namely the number of fulfilments of the norm that did not lead to undesired MAS states, $m_{H F}(n, t, C)$ is the number of harmful fulfilments of norm $n$ at time $t$, namely the number of fulfilments that led to undesired states, and $w_{S F}>0, w_{H F}>0$ weigh the importance of successful and harmful fulfilments of $n$ respectively. Notice that functions $m_{H F}$ and $m_{S F}$ receive as a parameter the set of undesired states $C$ so to assess if a norm fulfilment has lead to an undesired state or not. As an example, we may decide that a harmful fulfilment (that is, a norm fulfilment that leads to an undesired state), must be punished with a (negative) reward much higher than the (positive) reward that may obtain a successful fulfilment.

We notice therefore that our approach is akin to reinforcement learning [Sutton and Barto 1998] since both the effectiveness and necessity of a norm $\left(\mu_{e f f}, \mu_{n e c}\right)$ are somehow learned by continuously aggregating rewards that have been computed from the outcomes of agents' norm compliance in the MAS. 
Analogously, we compute the necessity of norm $n$ up to time $t$ as:

$$
\mu_{n e c}(n, t, C)=(1-\beta) \times \mu_{n e c}(n, t-1, C)+\beta \times r_{n e c}(n, t, C)
$$

where $\mu_{n e c}(n, t-1, C)$ is the necessity of $n$ up to time $t-1 ; 0 \leq \beta \leq 1$ is a learning rate; and $r_{n e c}(n, t, C)$ is a reward value based on the harmful infringements of norm $n$ at time $t$. As well as with the effectiveness, the necessity of a norm at an initial time $t_{0}$ $\left(\mu_{n e c}\left(n, t_{0}, C\right)\right)$ is set to an initial constant value $k \in[0,1]$. The reward of an infringed norm is computed as:

$$
r_{n e c}(n, t, C)=\frac{w_{H I} \times m_{H I}(n, t, C)}{w_{H I} \times m_{H I}(n, t, C)+w_{S I} \times m_{S I}(n, t, C)}
$$

where $m_{H I}(n, t, C)$ is the number of harmful infringements of norm $n$, namely infringements that led to undesired states, $m_{S I}(n, t, C)$ is the number of successful infringements of norm $n$, namely those infringements that did not lead to undesired states, and $w_{H I}>0, w_{S I}>0$ weigh the importance of harmful and successful infringements of $n$ respectively. Moreover, IRON computes the effectiveness and necessity ranges of each norm during a time period $T_{w}$ (i.e., a time window to compute the performance ranges of each norm. These ranges will be essential (as we show below) to perform generalisations and specialisations. We assess the effectiveness $(\check{E})$ and necessity $(\check{N})$ ranges for each norm as follows:

$$
\begin{gathered}
\check{E}=\left[\bar{\mu}_{e f f}\left(n, T_{w}, C\right)-\hat{\mu}_{e f f}\left(n, T_{w}, C\right), \bar{\mu}_{e f f}\left(n, T_{w}, C\right)+\hat{\mu}_{e f f}\left(n, T_{w}, C\right)\right] \\
\check{N}=\left[\bar{\mu}_{n e c}\left(n, T_{w}, C\right)-\hat{\mu}_{n e c}\left(n, T_{w}, C\right), \bar{\mu}_{n e c}\left(n, T_{w}, C\right)+\hat{\mu}_{n e c}\left(n, T_{w}, C\right)\right]
\end{gathered}
$$

where $\bar{\mu}_{\text {eff }}\left(n, T_{w}\right)$ and $\hat{\mu}_{e f f}\left(n, T_{w}\right)$ stand for the average and deviation of the effectiveness of $n$ within $T_{w}$, respectively, and $\bar{\mu}_{n e c}\left(n, T_{w}\right)$ and $\hat{\mu}_{n e c}\left(n, T_{w}\right)$ stand for the average and deviation of the necessity of $n$ within $T_{w}$, respectively.

\subsection{Operators for normative networks}

IRON will search for a normative system that solves the NSP by transforming an initial normative network over time, hence moving from one normative system to another. With this aim, our norm synthesis mechanism implements a collection of normative network operators. Each operator transforms IRON's normative network $\left\langle\mathcal{N}, \mathcal{R}_{G}, \Delta, \delta\right\rangle$ into another one $\left\langle\mathcal{N}^{\prime}, \mathcal{R}_{G}^{\prime}, \Delta, \delta^{\prime}\right\rangle$. More specifically, IRON implements the following operators:

- The addition of a new norm into the normative system. As Table $\mathrm{I}$ formally specifies, whenever a new norm is synthesised, the add operator extends IRON's normative network with norm $n\left(\mathcal{N}^{\prime}=\mathcal{N} \cup\{n\}\right)$, and sets its state to active $\left(\delta^{\prime}(n)=\right.$ active $)$.

- The deactivation of a norm in the normative system. The implementation of this deactivate operator sets the state of a given norm to inactive. Hence, although the norm remains in the normative network, it is no longer part of the normative system.

- The generalisation of a set of norms in the normative system into a more general norm (e.g., considering the example in Section 2, generalising $n_{1}, n_{2}$, and $n_{3}$ into $n_{5}$ ). As Table 1 shows, this generalise operator generalises a set of norms (children) into a more general norm (parent) by: (i) adding the parent norm to the network; (ii) establishing new generalisation relations $\left(R_{G}\right)$ between each generalised (child) norm and the parent norm in the normative network; (iii) setting the state of the parent to active and the children's to inactive. As a result, the child norms will no longer belong to the normative system, while the parent norm will.

- The specialisation of a norm in the normative system into more specific norms. This operation reverses the result of a generalisation (e.g., $n_{5}$ can be specialised into $n_{1}, n_{2}$, 
and $n_{3}$ ). Specifically, this specialise operator undoes the result of a generalisation by setting to inactive the state of the parent (more general) norm and setting to active the state of its children. Thus, thereafter all the child norms become candidates to belong to the normative system, while the parent norm does not any longer.

Table I. IRON operators.

\begin{tabular}{l|l}
\hline Operator & Specification \\
\hline add $(N N, n)$ & $\mathcal{N}^{\prime} \leftarrow \mathcal{N} \cup\{n\}$ \\
& $\delta^{\prime}(n) \leftarrow$ active \\
& $N N^{\prime} \leftarrow\left\langle\mathcal{N}^{\prime}, R_{G}, \Delta, \delta^{\prime}\right\rangle$ \\
\hline deactivate $(N N, n)$ & $\delta^{\prime}(n) \leftarrow$ inactive \\
& $N N^{\prime} \leftarrow\left\langle\mathcal{N}, R_{G}, \Delta, \delta^{\prime}\right\rangle$ \\
\hline generalise $(N N$, parent, children $)$ & $\mathcal{N}^{\prime} \leftarrow \mathcal{N} \cup\{$ parent $\}$ \\
& $R_{G}^{\prime} \leftarrow R_{G} \cup\{($ ch, parent $) \mid$ ch $\in$ children $\}$ \\
& $\delta^{\prime}($ parent $) \leftarrow$ active \\
& $\delta^{\prime}($ ch $\leftarrow$ inactive for all ch $\in$ children \\
& $N N^{\prime} \leftarrow\left\langle\mathcal{N}^{\prime}, R_{G}^{\prime}, \Delta, \delta^{\prime}\right\rangle$ \\
\hline specialise(NN, parent, children $)$ & $\delta^{\prime}($ parent $) \leftarrow$ inactive \\
& for all child $\in$ children \\
& if $($ child, parent $) \in R_{G}$ \\
& $\delta^{\prime}($ child $) \leftarrow$ active \\
& $N N^{\prime} \leftarrow\left\langle\mathcal{N}, R_{G}, \Delta, \delta^{\prime}\right\rangle$ \\
\hline
\end{tabular}

\subsection{A strategy to synthesise normative systems}

Algorithm 2 describes in outline IRON's overall norm synthesis strategy. Since IRON is an on-line mechanism, at every tick it runs its strategy to perform the same three main tasks of the BASE algorithm (Algorithm 1 in Section 3), namely: (i) norm generation; (ii) evaluation of the current normative system; and (iii) refinement of the normative system. However, IRON implements them differently, thus, for example, refinement is done by means of specialisations, generalisations and deactivation of norms. Once the strategy finishes, it returns the normative system represented by the normative network.

\section{ALGORITHM 2: IRON strategy}

Input: $\left\langle s_{t-1}, s_{t}\right\rangle, N N, \mathcal{G}, f_{\text {apply }}, f_{\text {conflict }}, \mu_{\text {eff }}, \mu_{\text {nec }}, \Theta, T_{w}$

Output: $\Omega$

[1] conflictDescription $\leftarrow$ conflictDetection $\left(s_{t-1}, s_{t}, f_{\text {conflict }}\right)$;

[2] $\quad N N \leftarrow$ normGeneration $(N N$, conflictDescription, $\mathcal{G})$;

[3] $\quad P \leftarrow \operatorname{normEvaluation}\left(N N,\left\langle s_{t-1}, s_{t}\right\rangle, f_{\text {apply }}, f_{\text {conflict }}, \mu_{\text {eff }}, \mu_{n e c}, T_{w}\right)$;

[4] $\quad N N \leftarrow$ normRefinement $(N N, P, \mathcal{G}, \Theta)$;

[5] $\Omega \leftarrow\left\{n \in N N \mid \delta^{\prime}(n)=\right.$ active $\}$;

$[6]$ return $\Omega$

Algorithm 2 specifies IRON's general strategy (П). It is domain-independent and takes as input: (i) a pair $\left\langle s_{t-1}, s_{t}\right\rangle$ containing descriptions of the system state at time $t-1$ and time $t$, respectively. This pair stands for a transition between the system state at consecutive times. In fact, the differences between $s_{t-1}$ and $s_{t}$ reflect the local changes that occurred when the system evolved from $t-1$ to $t$; (ii) a normative network $N N$, which includes the current normative system $\Omega$; (iii) a grammar $\mathcal{G}$, including the subsumption relationships between its terms; (iv) a function $f_{a p p l y}$ to check norm applicability in the current system state $s$; (v) a function $f_{\text {conflict }}$ to detect if a given 
system state $s$ is undesired; (vi) two evaluation functions $\mu_{e f f}, \mu_{n e c}$ to assess the effectiveness and necessity of norms in $N N$; (vii) $\Theta$, a set of satisfaction degree thresholds described below (see Equations 8, 9, 10 and 11) $\left(\Theta=\left\{\alpha_{e f f}^{g e n}, \alpha_{n e c}^{g e n}, \alpha_{e f f}^{e s p}, \alpha_{n e c}^{e s p}\right\}\right)$; and (viii) a time period $T_{w}$.

The strategy is on-line and conflict-driven (since it is aimed to avoid undesired, conflicting states), and thus, at every tick, it starts by searching for conflicts in the current system state. Function conflictDetection (line 1) uses the domain-dependent function $f_{\text {conflict }}$ to assess if the current system state $s_{t}$ is undesired (i.e., it detects undesired states $C \subset S$ ). In case that $s_{t} \in C$, it returns a conflictDescription that incorporates descriptions $s_{t-1}$ and $s_{t}$, together with the identifiers of those agents whose actions lead to the undesired state $s_{t}$ (for instance, in a traffic scenario, those cars that went forward before colliding). Next, the normGeneration function (line 2) synthesises a norm to avoid the transition from $s_{t-1}$ to $s_{t}$ (though disregarding the generation of general norms) in order to avoid it in the future. Subsequently, normEvaluation in line 3 evaluates norms in terms of their effectiveness and necessity as discussed in Section 4.3. Finally, the norm refinement function in line 4 generalises and/or specialises norms according to their effectiveness and necessity ranges during the time period $T_{w}$, as discussed in Sections 4.6 and 4.7, below. The algorithm outputs a normative system (line 6 ) for the agents in the domain that IRON is aiming at regulating.

The main functions in IRON's strategy (i.e., in Algorithm 2) are specified in Algorithms 3, 4 and 5. As previously mentioned, our proposal is to monitor the evolution of the NMAS at regular time intervals (i.e., ticks) and apply operators under certain conditions. Next, we present how previously defined operators are invoked in the three main functions, which are specified as follows.

ALGORITHM 3: normGeneration

Input: $N N$, conflictDescription, $\mathcal{G}$

Output: $N N$

[1] if isEmpty(conflictDescription) then

[2] return $N N$

end

[3] $n \leftarrow$ generateNorm $(\mathcal{G}$, conflictDescription $)$;

[4] $N N \leftarrow \operatorname{add}(N N, n)$;

[5] potential $[n] \leftarrow$ potentialGeneralisations $(n, \mathcal{G})$;

[6] return $N N$

ALGORITHM 4: normEvaluation

Input: $N N,\left\langle s_{t-1}, s_{t}\right\rangle, f_{\text {apply }}, f_{\text {conflict }}, \mu_{\text {eff }}, \mu_{\text {nec }}, T_{w}$

Output: $P$

[1] applicableNorms $\leftarrow$ normApplicability $\left(N N,\left\langle s_{t-1}, s_{t}\right\rangle, f_{\text {apply }}\right)$;

[2] $(F, I) \leftarrow$ normCompliance $\left(\right.$ applicableNorms, $\left.f_{\text {conflict }}\right)$;

[3] for $n \in \operatorname{norms}(F, I)$ do

[4] $U[n] \leftarrow$ updateUtilities $\left(n, F, I, \mu_{\text {eff }}, \mu_{\text {nec }}\right)$;

[5] $\quad(\check{E}[n], \check{N}[n]) \leftarrow$ updatePerformanceRanges $\left(n, U[n], T_{w}\right)$;

[6] $\quad P[n] \leftarrow(\check{E}[n], \check{N}[n])$

end

[7] return $P$ 


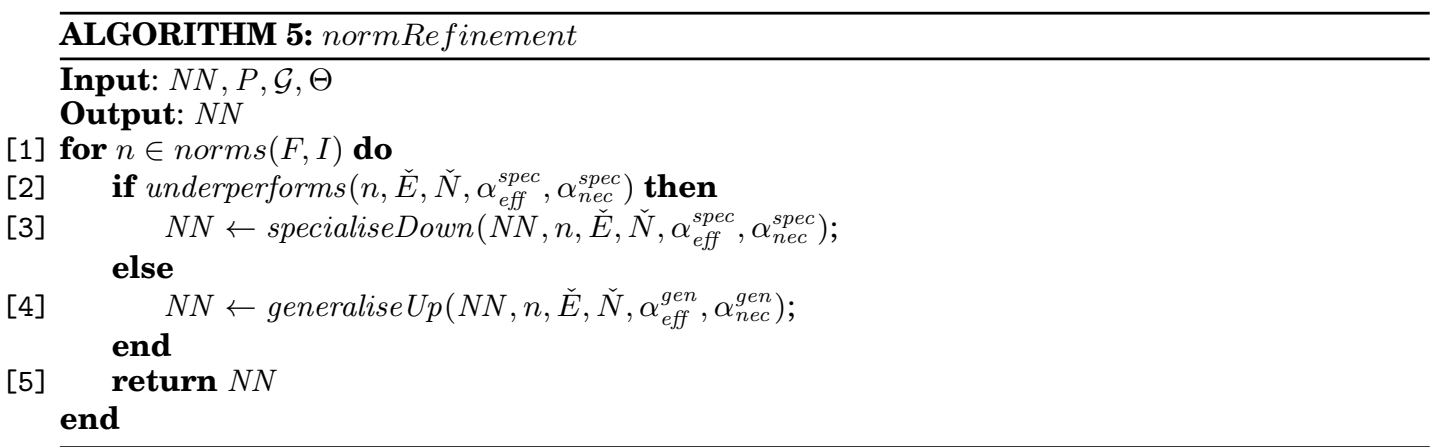

4.5.1. Generation of new norms. As mentioned before, IRON starts by detecting if the current system state $s_{t}$ is undesired or not. Function conflictDetection (line 2 in Algorithm 2) uses function $f_{\text {conflict }}$ to assess if $s_{t}$ belongs to the set of undesired states $C, s_{t} \in C$. Afterwards, if $s_{t}$ is an undesired state, the norm generation function (line 2 in Algorithm 2, and Algorithm 3) synthesises a new norm in order to avoid the transition from $s_{t-1}$ to $s_{t}$ in the future. Specifically, it firstly invokes function generateNorm (line 3 in Algorithm 3), which employs the unsupervised CBR mechanism and the grammar $\mathcal{G}$ together with subsumption relationships of its terms (see Section 4.1) to generate a new norm aimed to avoid the transition from $s_{-1}$ to $s_{t}$. Secondly, as line 4 in Algorithm 3 indicates, the newly created norm $n$ is added to the normative network by invoking the add operator. Thirdly, the norm generation function ends by creating all potential generalisations (see Algorithm 6 in Section 4.6) for each newly created norm $n$ (line 5).

Basically, the implementation of this generateNorm function is the same as for the BASE approach described in Section 3][Morales et al. 2011c]. The only difference is that our synthesis process does not generate general norms, since the generalisation process runs separately from norm generation. generateNorm is based on an unsupervised Case-Based Reasoning (CBR) approach. Hence, this approach is based on the following principle: if we can prevent a conflict in a given situation by enacting a norm, it is likely that we can prevent a conflict in a similar situation by means of a similar norm. Initially, when no other knowledge is available, norms are created based on the following heuristic: the transition to an undesired state is caused by the actions of the agents involved in that undesired state. Therefore, our generateNorm chooses one of the agents involved in an undesired state $s_{t}$ and gathers its context and performed action in $s_{t-1}$. Then, the norm is synthesised so that this action is prohibited for any agent encountering this same context. This is done with the aim of avoiding the previously resulting conflict in future situations. Obviously, there is no certainty about the validity of this norm, and this is the reason IRON needs to continuously evaluate it in the NMAS.

Our norm synthesis uses ground atomic formulae from the states, and focusses on a specific agent to figure out its context $\varphi$ (what it was able to perceive, given its position in the grid) and the action $a c$ it carried out (which possibly led to a conflicting state). The synthesised norm $\langle\varphi, \operatorname{prh}(a c)\rangle$ is thus made up of fully instantiated, non-negated atomic formulae, establishing the specific context of one agent and its action which led to a conflicting state. This process differs from induction of logical theories (e.g., inductive logic programming [Muggleton and Raedt 1994; Nienhuys-Cheng and Wolf] 1997]) in two important ways: $i$ ) the synthesised norm is specific with fully ground predicates (so we do not need to perform inverse resolution to synthesise norms with 
variables); ii) negated atoms do not play any role, as these are not represented in states.

Note that implicit within our model is a reduction of deontic operators to prohibitions that forbid actions in certain conditions. It is natural to ask whether this represents a problematic restriction. But, notice that obligations and permissions are frequently and naturally interpreted as dual notions: an action is obligatory if it is not permitted to refrain from performing the action; and an action is permissible if it is not obligatory to refrain from performing the action. In this case, obligations can be reduced to prohibitions: to make an action obligatory, prohibit everything else. With this in mind, our approach of focussing on prohibitions can be seen also encompassing obligations. Of course, richer deontic operators (e.g., conditional obligations) have also been considered in the literature, and we do not claim that our approach encompasses a full range of these: but with respect to core operators, our approach is sufficient.

Moreover, our approach can also handle permissions. We observe that the interpretation of permissions can vary. For instance, we may have normative systems whereby all actions are prohibited unless they are explicitly permitted. Alternatively, we may have systems whereby all actions are permitted unless explicitly prohibited, and permissions are ways of encouraging certain behaviours. When dealing with obligations and permissions, the undesired states are idealised situations which did not occur, and the context of an agent is used to establish an obligation or permission on a missing action (which would have reached the idealised situation).

4.5.2. Norm evaluation. As previously mentioned, the strategy updates the effectiveness and necessity of norms by evaluating them individually. In general, IRON evaluates a norm depending on the outcome (conflicts) that either its fulfilment or infringement lead to. Therefore, norm evaluation will solely consider the applicable norms that have been either fulfilled or infringed by the agents in the NMAS in the transition from two consecutive states in time.

The norm evaluation function is specified by Algorithm 4 . Function normApplicability (line 1) uses function $f_{\text {apply }}$ to assess the norms in the normative network $(N N)$ that were applicable at tick $t-1$. Thus, this function assesses the norms that are applicable at state $s_{t-1}$. Next, function normCompliance (line 2) partitions the selected applicable norms into fulfilled and infringed norms ( $F$ and $I$ ). Moreover, it uses a conflictdetection function $\left(f_{\text {conflict }}\right)$ to determine which norms led to undesired states. As a result, we obtain a partition of applicable norms into four multi-sets (sets that allow duplicate values): (i) fulfilled norms that led to undesired MAS states $\left(F_{C}\right)$; (ii) fulfilled norms that did not lead to undesired MAS states $\left(F_{\bar{C}}\right)$; (iii) infringed norms that led to undesired MAS states $\left(I_{C}\right)$; and (iv) infringed norms that did not lead to undesired MAS states $\left(I_{\bar{C}}\right)$. In the algorithm $F=\left(F_{C}, F_{\bar{C}}\right)$ and $I=\left(I_{C}, I_{\bar{C}}\right)$. These sets are the ones used by function updateUtilities (line 4 ) to compute the effectiveness and necessity of each norm at time $t$. In fact, this function implements the equations 2 |5 given in Section 4.3. Next, in line 5, function updatePerformanceRanges computes its effectiveness and necessity ranges during a period of time $T_{w}$ (see equations 6 and 7 of Section 4.3.).

4.5.3. Normative system refinement. The final function in our strategy is the normative system refinement, which yields a new normative system by transforming the normative network via specialisations and generalisations. Specifically, it specialises any under-performing norms (lines 2-3 in Algorithm 5) while it tries to generalise those norms that performed well (line 4). With this aim, the strategy keeps track of effectiveness and necessity ranges of the norms in the normative network during a period of time $T_{w}$. Then, the refinement task amounts to implementing the following rules: 


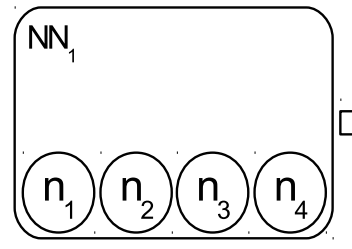

(a)

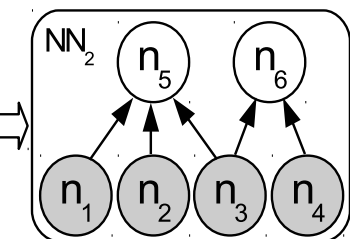

(b)

Fig. 2. Generalisation of $n_{1}, n_{2}, n_{3}, n_{4}$ : norms $n_{5}$ and $n_{6}$ are active, while the rest are inactive, so the normative system consists of $\Omega=\left\{n_{5}, n_{6}\right\}$.

- A norm is specialised (or deactivated if it has no children in the normative network) provided that either its effectiveness or necessity have not been good enough during $T_{w}$. This occurs when the effectiveness or necessity of some of its children have not been good enough either.

- A set of norms are generalised provided that: (i) they all relate to the very same norm (parent) in the normative network; (ii) they are all the possible child norms of the parent norm; (iii) their effectiveness and necessities have all been sufficiently high during $T_{w}$.

The following subsections describe both processes in more detail.

\subsection{Norm generalisation}

Norm generalisation starts whenever IRON detects some norm that has performed well during a period of time $T_{w}$. We say that a norm $n$ has performed well during a period $T_{w}$ if the lower bounds of its effectiveness and necessity ranges are above some satisfaction (generalisation) thresholds. This amounts to satisfying the following generalisation conditions:

$$
\begin{aligned}
& \check{E}_{\min }\left(n, T_{w}, C\right)>\alpha_{\text {eff }}^{g e n} \\
& \check{N}_{\text {min }}\left(n, T_{w}, C\right)>\alpha_{n e c}^{g e n}
\end{aligned}
$$

where $\check{E}_{\text {min }}\left(n, T_{w}, C\right)$ and $\check{N}_{\text {min }}\left(n, T_{w}, C\right)$ are the lower bounds of $\check{E}$ and $\check{N}$ that are described in equations 6 and $7 ; \alpha_{e f f}^{g e n} \in[0,1]$ and $\alpha_{n e c}^{g e n} \in[0,1]$ are the generalisation thresholds for effectiveness and necessity.

Given a generalisable norm, the generalisation process is based on building all potential generalisations for the norm to subsequently analyse whether each one of them can be enacted or not. If a potential generalisation is enacted, it transforms the current normative system into another one. Otherwise, the normative system remains unchanged. Next, we illustrate: (i) how to build potential generalisations; and (ii) how to enact potential generalisations. We illustrate our approach with norms $n_{1}-$ $n_{6}$ from Section 2, their effectiveness and necessity ranges listed in Table II] and $\alpha_{e f f}^{g e n}=0.5, \alpha_{n e c}^{g e n}=0.4$ as generalisation thresholds.

4.6.1. How to build potential generalisations. We now consider norm $n_{3}$ described in Section 2. The first step to build a potential generalisation is to find a more general parent norm. With this aim, our generalisation process employs a grammar $\mathcal{G}$ together with subsumption relationships of its terms (see Section 4.1) to build predicates of the language $\mathcal{L}_{A g}$. In our example, with the aid of the grammar, $n_{5}$ would be built, which is more general than $n_{3}$ and thus can be a parent of a potential generalisation. The second step is to find all the children of the parent. In our example, the children of $n_{5}$, (the siblings of $n_{3}$ ), are $n_{1}$ and $n_{2}$. The triple $\left\langle n_{3}, n_{5},\left\{n_{1}, n_{2}\right\}\right\rangle$ records a potential generalisation, that is, $\left\langle n, n^{\prime}, S\right\rangle$, where $n$ is a generalisable norm, $n^{\prime}$ is a parent norm of 
Table II. $\check{E} \& \check{N}$ ranges for norms in Figure 2

\begin{tabular}{|c|c|c|}
\hline & $\check{E}$ & $\check{N}$ \\
\hline$n_{1}$ & {$[0.6,0.7]$} & {$[0.5,0.6]$} \\
\hline$n_{2}$ & {$[0.7,0.8]$} & {$[0.6,0.7]$} \\
\hline$n_{3}$ & {$[0.8,0.9]$} & {$[0.5,0.7]$} \\
\hline$n_{4}$ & {$[0.7,0.9]$} & {$[0.6,0.8]$} \\
\hline$n_{5}$ & {$[0.6,0.9]$} & {$[0.5,0.8]$} \\
\hline$n_{6}$ & {$[0.6,0.8]$} & {$[0.6,0.9]$} \\
\hline
\end{tabular}

$n$, and $S$ is a possibly empty set of any other norms which $n^{\prime}$ generalises (disregarding $n$ ). The generalisation process now continues to build all potential generalisations. In our example, the triple $\left\langle n_{3}, n_{6},\left\{n_{4}\right\}\right\rangle$ would be also built as a potential generalisation of $n_{3}$.

4.6.2. How to enact potential generalisations. A potential generalisation $\left\langle n, n^{\prime}, S\right\rangle$ can be enacted if, and only if, all the siblings of $n$ (the norms in $S$ ) belong to the normative network (that is, they have been previously created by the norm generation process) and satisfy all the generalisation conditions of equations 8 and 9 . If these conditions hold, the potential generalisation is enacted to transform the normative system. As an example, consider the two potential generalisations $\left\langle n_{3}, n_{5},\left\{n_{1}, n_{2}\right\}\right\rangle$ and $\left\langle n_{3}, n_{6},\left\{n_{4}\right\}\right\rangle$ and the normative network from Figure 2 a showing $n_{1}, n_{2}, n_{3}$ and $n_{4}$ as active norms (that is, we have a normative system $\Omega_{1}=\left\{n_{1}, n_{2}, n_{3}, n_{4}\right\}$ ). From Table II , we observe that norms $n_{1}, n_{2}, n_{4}$ fulfil the generalisation conditions in equations 8 and 9 , and so does $n_{3}$. Thus, the first potential generalisation can be enacted as follows: (1) norm $n_{5}$ is created, added to the normative network, and activated; (2) $n_{1}, n_{2}$ and $n_{3}$ are deactivated in the normative network; (3) generalisation relationships are established between $n_{1}, n_{2}, n_{3}$ and $n_{5}$. This amounts to applying the generalise $\left(N N, n_{5},\left\{n_{1}, n_{2}, n_{3}\right\}\right)$ operator in Section 4.4. Analogously, we can enact the second potential generalisation by invoking generalise $\left(N N, n_{6},\left\{n_{4}, n_{3}\right\}\right)$. Figure $2 \mathrm{~b}$ shows the resulting normative network after both norm generalisations. It contains two active norms $\left(n_{5}, n_{6}\right)$, and four inactive norms $\left(n_{1}, n_{2}, n_{3}\right.$, and $\left.n_{4}\right)$, that is, we have the normative system $\Omega_{2}=\left\{n_{5}, n_{6}\right\}$. These two norm generalisations reduce the size of the normative system from 4 norms to 2, and the total number of predicates from 12 to 6 (improving its compactness).

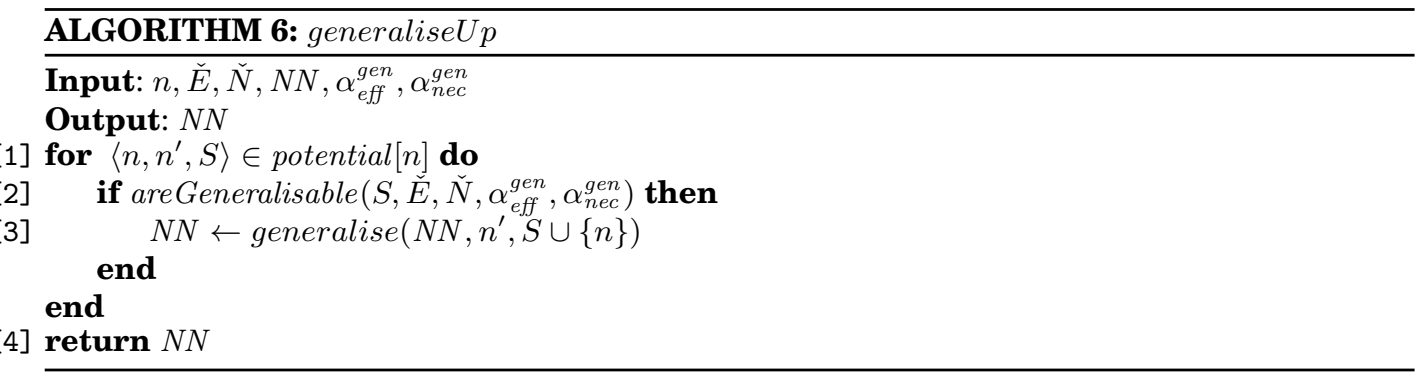

4.6.3. Generalisation algorithm. Norm generalisations are performed by the function generalise $U p$ in Algorithm 6. Its inputs are a norm to generalise $(n)$ along with its effectiveness and necessity ranges $(\tilde{E}, N)$, a normative network $(N N)$, and the thresholds to verify the generalisation conditions. For each potential generalisation of norm 


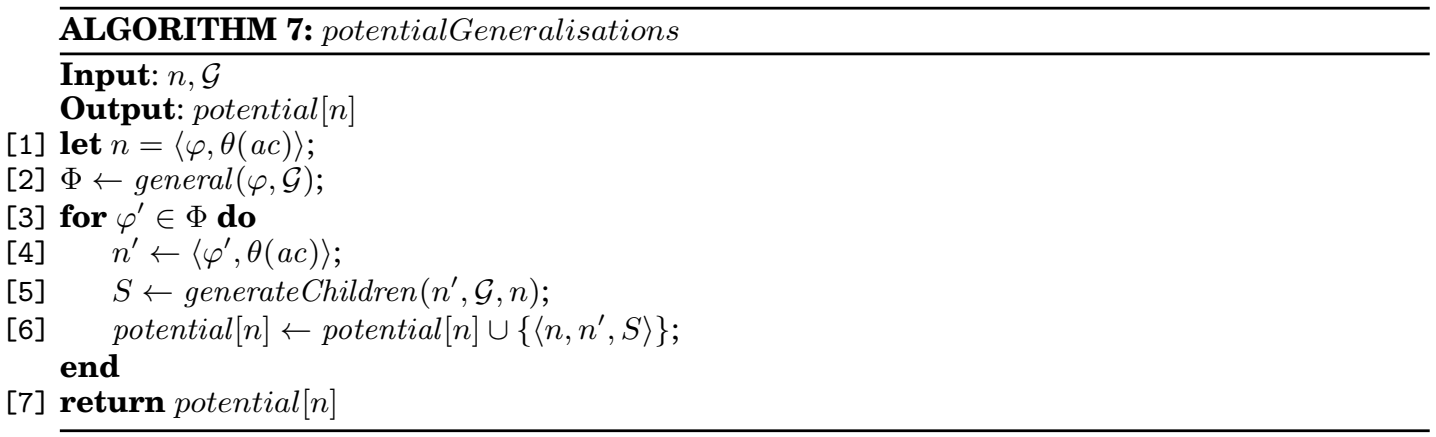

$n$ (line 1), function areGeneralisable (line 2) verifies whether all norms in $S$ (i.e, the children of the generalisation) satisfy the generalisation conditions. If so, the operator generalise $\left(N N, n^{\prime}, S \cup\{n\}\right)$ (line 3) adds the parent norm to the normative network $N N$ and activates it, deactivates the child norms, and establishes generalisation relationships between child norms and parent norm. Additionally, the auxiliary function potentialGeneralisations can be employed to generate all the potential generalisations for a given norm $n$ (indeed, as line 4 in Algorithm 3 shows, our strategy invokes this function when creating the norm). First, it takes the precondition $\varphi$ of a norm $n$ (line 1 ) and employs function general (line 2) to obtain its parent preconditions $\Phi$ by using grammar $\mathcal{G}$ together with subsumption relationships of the terms of $\mathcal{L}_{A g}$. Second, in lines 3-4, for each parent precondition $\varphi^{\prime} \in \Phi$ it builds $n^{\prime}$, a parent norm, based on the general precondition $\varphi^{\prime}$ and the same consequent $\theta(a c)$ from $n$ (that is, $n^{\prime}=\left\langle\varphi^{\prime}, \theta(a c)\right\rangle$ ). Next, function generateChildren $\left(n^{\prime}, \mathcal{G}, n\right)$ (line 5) computes (using again grammar $\mathcal{G}$ and subsumption term relationships) child norms of $n^{\prime}$ disregarding $n$. Finally, in line 6 , it builds a new potential generalisation $\left\langle n, n^{\prime}, S\right\rangle$ which is added to the potential generalisations of norm $n$.

\subsection{Norm Specialisation}

Norm specialisations make it possible to refine generalisations of norms that do not perform well during a period of time. Take the normative network depicted in Figure $3 \mathrm{a}$ with a single active norm, $\Omega_{1}=\left\{n_{10}\right\}$. The network contains norms $n_{1}, n_{2}, n_{3}$ and $n_{5}$ (defined in Section 2), together with:

$$
\begin{aligned}
& n_{7}:\langle\{l \text { left }(\text { car }), \quad \text { front }(\text { car }), \operatorname{right}(\text { nil })\}, \operatorname{prh}(\text { Go })\rangle \\
& n_{8}:\langle\{\text { left(bike), front }(\text { car }), \operatorname{right}(\text { nil })\}, \operatorname{prh}(G o)\rangle \\
& n_{9}:\langle\{\text { left(private),front (car), right(nil) }\}, \operatorname{prh}(G o)\rangle \\
& n_{10}:\langle\{l e f t(\text { vehicle }), \text { front }(\text { car }), \operatorname{right}(\text { nil })\}, \operatorname{prh}(G o)\rangle
\end{aligned}
$$

Norms $n_{7}$ and $n_{8}$ prohibit a car from proceeding whenever there is a car or a bike to its left, a car in front and nothing to its right. Norm $n_{9}$ is a generalisation of norms $n_{7}$ and $n_{8}$ to give way to private vehicles on the left, whereas norm $n_{10}$ is a generalisation of all norms from $n_{1}$ to $n_{9}$ to give way to any vehicle on the left. Recall from Section 4.1 that IRON uses a grammar to construct norms. Each norm contains a precondition with three predicates left, front, right, and a postcondition with a prohibition to perform action Go. Notice that all norms from $n_{1}$ to $n_{10}$ are constructed by means of this grammar. In Figure $3 a$, norm $n_{10}$ is the only active norm, and we assume that all the norms below $n_{10}$ have been previously generalised to $n_{10}$. 


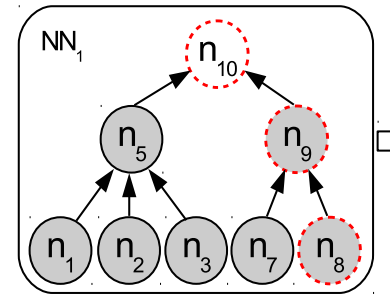

(a)

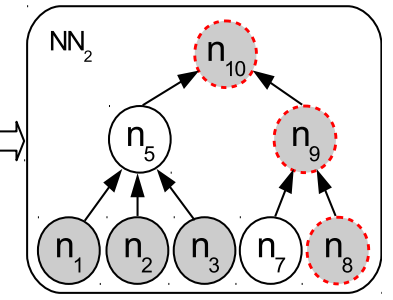

(b)

Fig. 3. Specialisation of norm $n_{10}$ into norms $n_{5}$ and $n_{7}$. a) $\Omega_{1}=\left\{n_{10}\right\}$, b) $\Omega_{2}=\left\{n_{5}, n_{7}\right\}$

Norm specialisation starts out whenever IRON detects that some norm has underperformed during a period of time $T_{w}$. We say that a norm $n$ has under-performed during a period $T_{w}$ if the higher bound of its effectiveness range or the higher bound of its necessity range are below the satisfaction (specialisation) thresholds provided in the strategy (see Section 4.5). This amounts to satisfying either (or both) of the two following conditions. $2^{2}$

$$
\begin{gathered}
\check{E}_{\text {max }}\left(n, T_{w}, C\right)<\alpha_{\text {eff }}^{\text {spec }} \\
\check{N}_{\text {max }}\left(n, T_{w}, C\right)<\alpha_{n e c}^{s p e c}
\end{gathered}
$$

where $\check{E}_{\max }\left(n, T_{w}, C\right)$ and $\check{N}_{\max }\left(n, T_{w}, C\right)$ are the higher bounds of $\check{E}$ and $\check{N}$ that are described in equations 6 and 7. Let us suppose that norms $n_{1}, n_{2}, n_{3}, n_{5}$ and $n_{7}$ have performed well during a period $T_{w}$, but norms $n_{8}, n_{9}$ and $n_{10}$ have not. This is a reasonable assumption. Since $n_{9}$ and $n_{10}$ are more general than $n_{8}$, they also apply in the same situations in which $n_{8}$ does. This means that the poor performance of $n_{8}$ affects $n_{9}$, whose performance affects in turn $n_{10}$. In general, the performance of a general norm is affected by the performance of the norms it generalises. Here, IRON would choose the most general norm, $n_{10}$, as the norm to specialise. The process would start by invoking operator specialise. This operator first deactivates $n_{10}$ and activates its child norms, $n_{5}$ and $n_{9}$. Next, IRON would attempt to specialise norms $n_{5}$ and $n_{9}$. Since norm $n_{5}$ has performed well, it would remain active. However, since norm $n_{9}$ has under-performed, it would be deactivated while its children $\left(n_{7}\right.$ and $\left.n_{8}\right)$ would be activated. Finally, the under-performing norm $n_{8}$ would be deactivated and, since it has no children (and $n_{7}$ performs well), the specialisation process would finish. The resulting network is the normative system $\Omega_{2}=\left\{n_{5}, n_{7}\right\}$ in Figure $3 \mathrm{~b}$.

4.7.1. Specialisation algorithm. In Algorithm 8 the function specialiseDown recursively specialises a norm $n$ into its children until it reaches the leaves of the network. If the norm is a leaf (line 1), it is deactivated so that it does not belong to the normative system (line 2). Otherwise, operator specialise (line 4) specialises $n$ by: (i) deactivating it; and (ii) activating all its children. Next, each under-performing child norm is specialised down in turn (lines 5-7). As a result, the algorithm refines a generalisation by deactivating those child norms that under-performed while keeping active those that performed well. Notice therefore that our specialisation algorithm makes it possible to perform a fine-grained backtracking over general norms that do not perform well.

${ }^{2}$ Notice that these conditions are highly conservative, since they require the higher value in the range for period $T_{w}$ to be below the threshold value. This is also the case for the generalisation conditions in equations 8 and 9 since in order to generalise the lower value in the range is required to be above the threshold. 


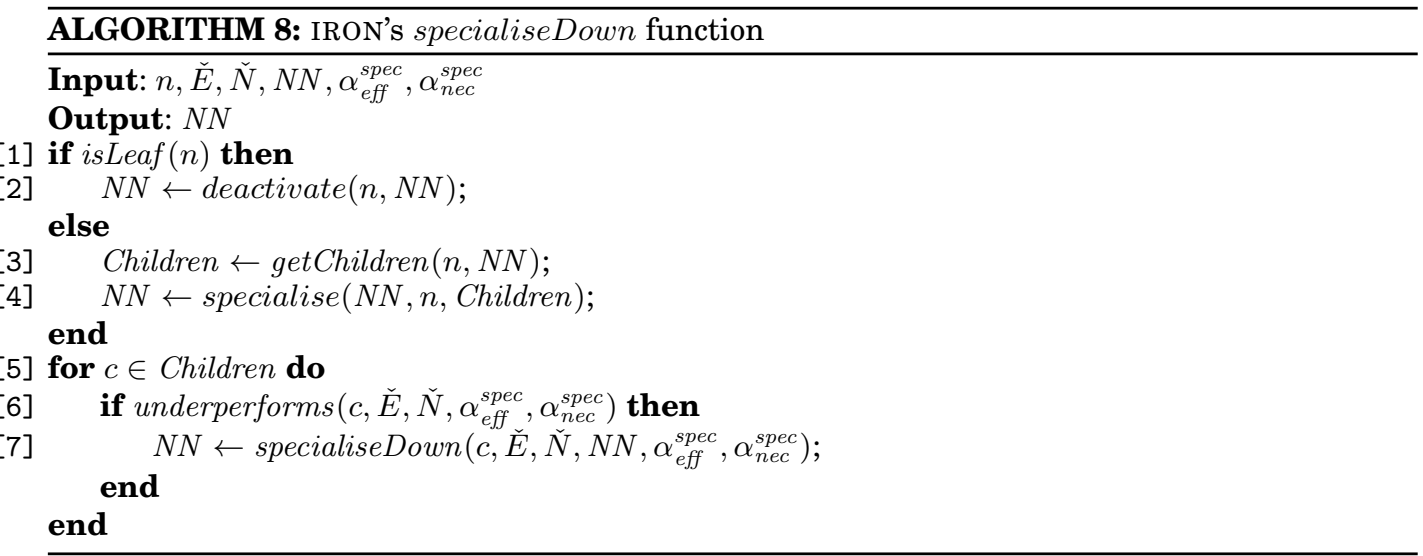

\subsection{Complexity analysis}

Finally we are ready to analyse the computational complexity of IRON. Before that, we will consider the number of norms that can be generated by a particular grammar $\mathcal{G}$. If $p$ is the maximum number of predicates of any norm generated by the grammar, $r$ is the maximum arity of any predicate, and $d$ is the maximum number of terms at any position of any given predicate, the number of norms that can be generated by grammar $\mathcal{G}$ is $d^{r \cdot p}$. Given a grammar $\mathcal{G}$ we shall note the number of norms it can generate as $\eta_{\mathcal{G}}$. Moreover, given a CBR base $C$, we shall note as $\eta_{C}$ the number of norms in the case base. Now we are ready to assess IRON's complexity.

LEMMA 4.1. The norm synthesis performed by the IRON algorithm when employing grammar $\mathcal{G}$ and case base $C$ when detecting $\kappa$ conflicts takes time $O\left(\kappa \cdot \eta_{C}+3 \cdot|A g|\right.$. $\left.|N N|+\eta_{\mathcal{G}}(|A g| \cdot|N N|+1)\right)$.

PRoOF. The norm generation stage involves (i) generating norms for all detected conflicts and (ii) generating all potential generalisations for each new norm. The cost of the first step is $O\left(\kappa \cdot \eta_{C}\right)$, whereas the cost of the second step is $O\left(\eta_{\mathcal{G}}\right)$. The cost of the norm evaluation process is $O(3 \cdot|A g||N N|)$, since it involves assessing the applicability of norms $(O(|A g||N N|))$, assessing the compliance with norms $(O(|A g||N N|))$, and updating norms' utilities and performance ranges $(O(|A g||N N|))$. Finally, the cost of norm refinement is $O\left(\eta_{\mathcal{G}} \cdot|A g||N N|\right)$, which amounts to the worst case cost of generalising norms, since the cost of specialising norms is $O\left(|A g||N N|^{2}\right)$. Putting all together, the resulting worst-case time is $O\left(\kappa \cdot \eta_{C}+3 \cdot|A g| \cdot|N N|+\eta_{\mathcal{G}}(|A g| \cdot|N N|+1)\right)$.

Observe that the computation time of IRON is larger than BASE. Nonetheless, as we will show in the experiments carried out in Section 5, this is the price paid by IRON in order to significantly outperform BASE in terms of compactness.

As a final remark, notice that given a grammar $\mathcal{G}$, the number of normative systems is $2^{\eta_{\mathcal{G}}}$. This is precisely the size of the search space that IRON must explore in search for compact normative systems. However, we recall that IRON is an approximate algorithm for norm synthesis, and it does not require exploring the whole search space, as we will demonstrate in Section 5 .

\subsection{Architecture and computational model}

We now have all the components of the architecture and computational model of our norm synthesis system. Figure 4 illustrates the overall architecture of IRON. 


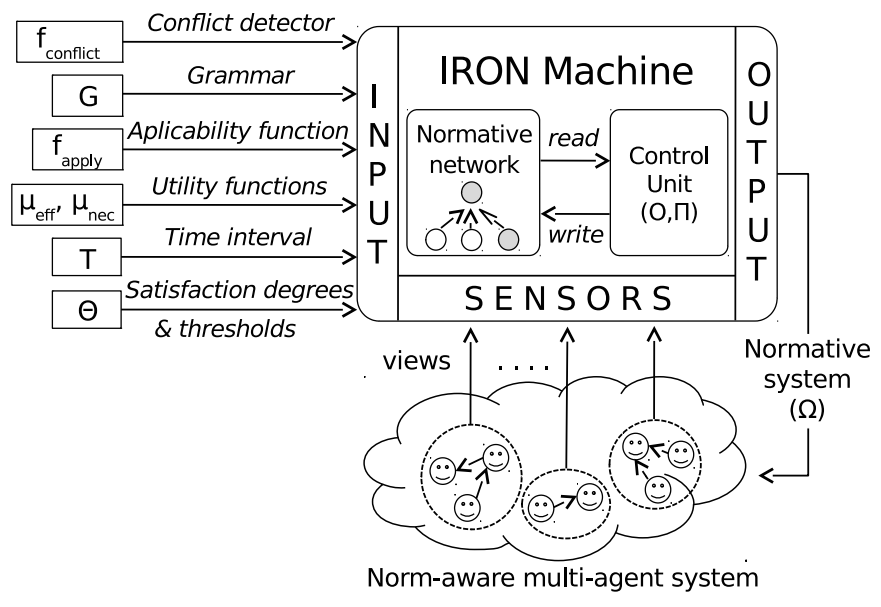

Fig. 4. IRON's architecture

As we mentioned, IRON continuously searches on-line for a solution to the norm synthesis problem, namely during the operation of a NMAS. We can thus regard IRON as an external observer of agents' interactions.

IRON has a domain independent abstract architecture which is composed of the following domain independent elements: (1) a data structure to represent explored norms, namely a normative network; (2) a set of operators to apply changes to the normative network; and (3) a general, abstract strategy to perform norm synthesis for any scenario. In order to perform norm synthesis for a given scenario, this domain independent machine receives as an input the following domain dependent elements: (i) a function ( $\left.f_{\text {conflict }}\right)$ to detect undesired states; (ii) a grammar $\mathcal{G}$ to define norms; (iii) a function to determine whether a norm applies to the agents in a given state $\left(f_{\text {apply }}\right)$; (iv) evaluation functions to compute the effectiveness $\left(\mu_{\text {eff }}\right)$ and necessity $\left(\mu_{\text {nec }}\right)$ of norms and normative systems; (v) the satisfaction degrees and thresholds $(\Theta)$; as well as (vi) the time interval $(T)$ considered when solving the NSP.

Our norm synthesis mechanism is composed of: (i) a normative network $(N N)$ to compactly represent the current normative system and to store the norms synthesised (explored) so far; (ii) a control unit in charge of directing the NSP solving. The control unit continuously perceives the target NMAS by describing the current MAS state. After that, the control unit calls the strategy $\Pi$ described in Section 4.5 to apply a collection of operators $O$ (see Section 4.4) and to eventually produce a new normative system that prevents the conflicts observed in the current system state.

The normative system $(\Omega)$ is broadcast to the agents in the NMAS. Once the new normative system is deployed, the control keeps on perceiving the MAS and generating descriptions of states of the NMAS to be analysed by the strategy. This cyclic process continues until the control unit receives from the strategy a normative system that is evaluated effective and necessary enough, according to the evaluation functions and satisfaction degrees set as input, during a period of time $T$. Such a normative system will represent a solution to the norm synthesis problem.

\section{EMPIRICAL ANALYSIS AND RESULTS}

In this section we present an empirical evaluation of the IRON norm synthesis mechanism. Section 5.1 describes the experimental settings whereas Section 5.2 provides results of the empirical evaluation; Sections 5.2.1 and 5.2.2 illustrate a stability anal- 
ysis and a convergence analysis of IRON, respectively. Finally, Section 5.2.3 compares IRON with BASE in terms of stability and compactness. We demonstrate that IRON is more stable than BASE and synthesises normative systems that are more compact than those synthesised by BASE.

\subsection{Empirical settings}

With the aim of comparing IRON with BASE, our experiments use the same traffic scenario than the one used in [Morales et al. 2011c]. Our experiments simulate a traffic junction composed of two orthogonal roads represented by a $20 \times 20$ grid (simulator available in the on-line appendix). Each road has two 20-cell lanes (one per direction).

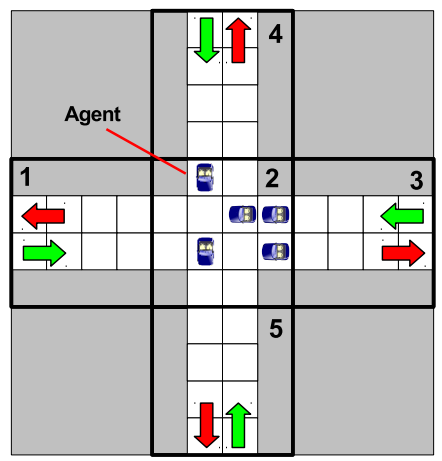

(a)

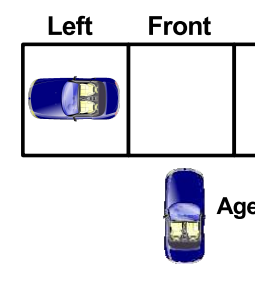

(b)

Fig. 5. a) Central area of the traffic junction. b) Agent context.

Figure $5 \mathrm{a}$ shows a $12 \times 12$ sub grid that corresponds to the centre of the junction. Each agent is a car that travels along the grid at one cell per tick by following a random trajectory. Specifically, each car enters the scenario from four possible start points (light/green points in Figure 5a), and travels towards randomly chosen destinations (exit points, depicted in dark/red in Figure 5 a). In this particular scenario, undesired states are those MAS states that contain collisions between cars. Thus, IRON will synthesise norms to avoid collisions. Since IRON is devoted to avoid undesired states, we consider a scenario that potentially leads to a large number of undesired states. Therefore, in order to favour a high frequency of collisions (i.e., undesired states), we use a high traffic density (from $41 \%$ to $48 \%$ of occupied cells) by having three cars entering the scenario every tick. At each tick, each car decides whether to comply or not with the norms output by IRON according to a probability, namely a norm infringement rate. The norm infringement rate is fixed at the beginning of each simulation and is the same for all cars.

Each experiment consists of a set of different simulations. IRON starts each simulation with an empty normative system ${ }^{3}$, As the simulation progresses, collisions among cars occur, and IRON then synthesises normative systems to avoid future collisions. The simulation finishes whenever it reaches 50,000 ticks or IRON converges to a stable normative system, hence removing undesired states and solving the norm synthesis problem (NSP). We assume that IRON has converged to a normative system if during a 10,000-tick period: (i) the normative system remains unchanged; and (ii) no new (non-regulated) undesired MAS states are detected. Non-regulated conflicts are those undesired MAS states that have not arisen from previous norm infringements.

\footnotetext{
${ }^{3}$ Notice however that IRON can also start operating with a non-empty normative system
} 
That is, no agent infringed a norm during the transition from the previous state to the undesired state. We assume that an undesired state that arises from a norm infringement is a regulated conflict (that is, a previously arisen undesired state that triggered the generation of the currently infringed norm), and thus, it is not considered when assessing convergence. This assumption is based on the intuition that we cannot ensure that if the agents had fulfilled the norm, the undesired state would have arisen.

In order to generate norms, IRON receives as an input a grammar which is as follows. The precondition of each norm contains three predicates left, front, right, which represent the 3 cells in the context of a reference car (see Figure 5b). Each predicate contains one term out of a set of six terms \{car-heading-left, car-heading-right, car-opposite-heading, car-same-heading, nil, anything\}. The first four terms represent a car along with the direction it is heading to, while term nil represents no car and term anything represents both a car or nothing. Furthermore, the following subsumption relationships for the terms in the grammar hold: car-heading-left $\sqsubseteq$ anything, car-heading-right $\sqsubseteq$ anything, car-opposite-heading $\sqsubseteq$ anything, car-same-heading $\sqsubseteq$ anything, nil $\sqsubseteq$ anything. The postcondition of each norm uses only prohibitions $(\theta=p r h)$ over an action. The actions available to agents are $A c=\{G o, S t o p\}$. Nevertheless, in this particular setting car agents just perform action Stop whenever a norm prohibits them to go forward. Therefore, the grammar that we employ can synthesise $6^{3}=216$ different norms and the number of normative systems to consider amounts to $2^{216}\left(>10^{65}\right)$. As an example, in our experiments IRON generates norms of the form:

$n:\langle\{$ left(car-heading-right),front(car-heading-right),right(car-heading-right $\}, \operatorname{prh}($ Go $)\rangle$

$$
n^{\prime}:\langle\{\text { left(car-heading-right), front(car-heading-right), right }(\text { nil })\}, \operatorname{prh}(G o)\rangle
$$

Norm $n$ prohibits a car from moving on (hence giving way) if the three cells in its context contain cars, each heading towards its right. Similarly, norm $n^{\prime}$ prohibits a car from going if the left and front cells in its context contain cars heading towards its right, and its right cell contains nothing. Notice that both norms $n, n^{\prime}$ only differ in their right position.

Regarding IRON's configuration parameters, we have taken a conservative approach to set them. This decision is intended to refine the normative system only when norms are slightly effective or necessary. We set IRON's parameters as follows: (i) low deactivation thresholds $\left(\alpha_{\text {eff }}^{\text {spec }}=0.2, \alpha_{\text {nec }}^{\text {spec }}=0.2\right.$ ) to only deactivate norms performing very poorly; (ii) high generalisation thresholds $\left(\alpha_{\text {eff }}^{\text {gen }}=0.6, \alpha_{\text {nec }}^{\text {gen }}=0.4\right)$ to only generalise norms when performing very well; (iii) weights in equation (3) $w_{A_{C}}=5$ and $w_{A_{\bar{C}}}=1$ to ensure that norm fulfilments leading to collisions (ineffective norms) are much more penalised than those avoiding collisions (effective norm); (iv) weights in equation (5) $w_{H_{I}}=2$ and $w_{S_{I}}=1$ to ensure that norm infringements leading to collisions (harmful infringements, necessary norm) obtain a much higher reward than those leading to no collisions (successful infringements, unnecessary norm). Additionally, we compute norms' effectiveness and necessity range over a long period of $\left|T_{w}\right|=200$ different norm evaluations, and norms effectiveness and necessity values, namely $\mu_{e f f}, \mu_{n e c}$, are initially set to a value $k=0.5$.

\subsection{Empirical results}

We now analyse the results of our empirical evaluation. First, we perform a stability analysis of IRON. We perform a micro analysis to study how IRON manages to solve the norm synthesis problem, hence removing new collisions (i.e., non-regulated collisions) in our traffic scenario. Additionally, we perform a macro analysis that shows that IRON is able to converge despite a large proportion of non-compliant behaviours 
in the overall agent population. Second, we compare IRON with BASE in terms of stability and compactness. We show that IRON is more stable than BASE, since it converges for greater norm infringement rates than BASE. Moreover, IRON manages to synthesise more compact normative systems than those synthesised by BASE, decreasing the overall number of norm predicates in the normative system.

5.2.1. Stability Analysis of IRON. We present a stability analysis to show how IRON manages to successfully synthesise normative systems that solve the norm synthesis problem. We compute the stability degree as a convergence rate, namely the number of simulations that converged to a stable normative system out of the total number of simulations. We notice that since undesired states trigger norm synthesis, the lack of collisions causes the normative system to remain stable.

We performed a simulation to show IRON's convergence process. In particular, Figure 6 illustrates the normative changes (i.e., the time steps in which the the normative network and/or the normative system change) for a single simulation with 0.3 norm infringement rate. That is, on average, agents decide to infringe norms 3 out of 10

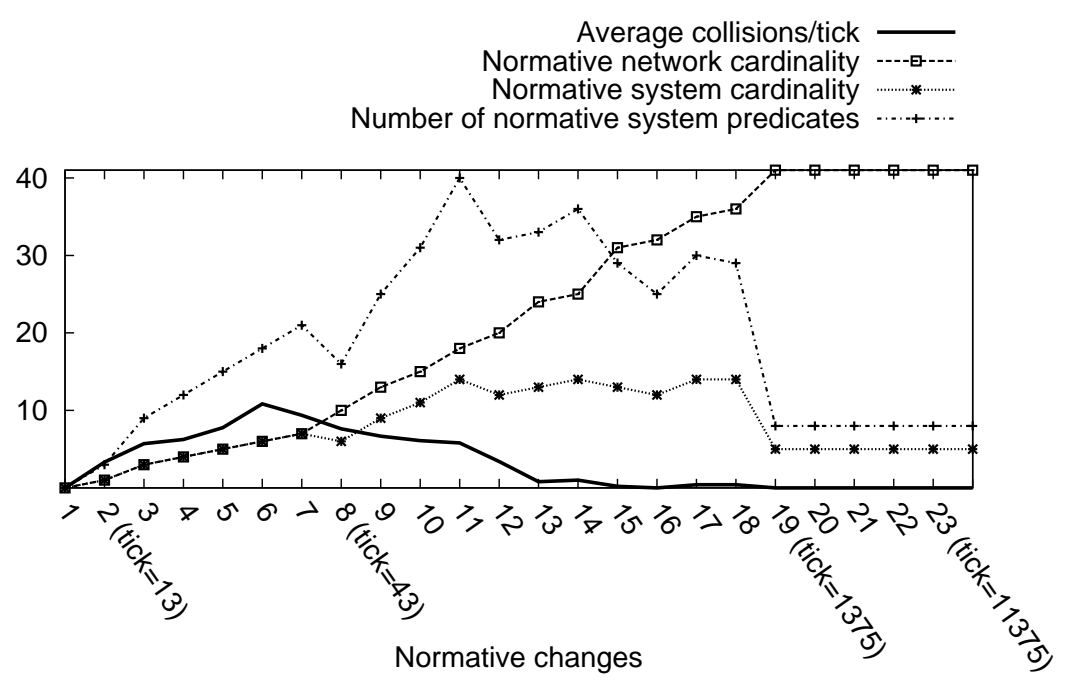

Fig. 6. Norm synthesis along a single simulation. The x-axis corresponds to normative changes (i.e., changes in the normative network and/or the normative system), and the y-axis corresponds to the different measures in the legend.

times, which can be considered as a high enough norm infringement rate. This figure shows: (i) the average of new car collisions (i.e., collisions that are not caused by norm infringements) per tick along time; (ii) the normative network cardinality, namely the total number of synthesised norms; (iii) the cardinality of the normative system, namely the number of active norms in the normative network; and (iv) the number of predicates in the normative system. At tick 13 (which corresponds to the 2nd normative change), the first collision arises and IRON synthesises the first norm. From that tick onwards, IRON keeps generating norms when needed, hence increasing the cardinality of both the normative network and the normative system. As a consequence, the number of predicates in the normative system increases as well. At tick 43 (8th normative change), IRON performs the first norm generalisation, reducing the 
cardinality of the normative system from 9 to 8 norms. As a result of this norm generalisation, the number of norms and predicates in the normative system decreases, thus increasing its compactness. Up to tick 1,375 (19th normative change), IRON keeps generating and generalising norms when possible. Norm generalisations reduce the total number of predicates of the normative system. At tick 1,375 IRON performs the last norm generalisation, hence synthesising a compact normative system of 5 norms with 8 predicates in total. From tick 1,375 onwards, the normative system remains stable. By using the resulting normative system, cars that comply with norms do not cause collisions. However, those cars may collide with other cars that infringe norms. Recall that those collisions that have arisen from norm infringements are not taken into account when assessing convergence. After 10,000 further ticks, IRON reaches the convergence criteria (tick 11,375 / 23th normative change). Overall, IRON explored 41 different norms (out of 125 possible ones), which were generalised into 5 norms, to find a 5-norm normative system that successfully prevents collisions as long as cars comply with norms.

Table III. A normative system upon convergence

\begin{tabular}{|c|c|c|c|c|}
\hline Norm & Pre-condition $(\theta)$ & Modality & $\bar{\mu}_{\text {eff }}$ & $\bar{\mu}_{\text {nec }}$ \\
\hline$n_{1}$ & left $($ car-heading-right) & $\operatorname{prh}(G o)$ & 0.86 & 0.90 \\
\hline$n_{2}$ & left(car-heading-left),front(car-heading-left $)$ & $\operatorname{prh}(G o)$ & 0.87 & 0.73 \\
\hline$n_{3}$ & front(car-heading-right),right(car-heading-right $)$ & $\operatorname{prh}(G o)$ & 0.86 & 0.81 \\
\hline$n_{4}$ & front(car-same-heading) & $\operatorname{prh}(G o)$ & 0.83 & 0.33 \\
\hline$n_{5}$ & front(car-heading-left $),$ right(car-heading-left $)$ & $\operatorname{prh}(G o)$ & 0.81 & 0.75 \\
\hline
\end{tabular}

The 5-norm normative system that IRON converged to is shown in Table III. Norm $n_{1}$ is a left-hand side priority norm specifying that a car must stop when it observes a car to its left which is heading to its right, and no matter what it perceives in front or to its right. It has very high values of effectiveness $(0.86)$ and necessity $(0.90)$, since it represents a situation that leads to collisions most of the times. Therefore, we consider this norm as an essential norm. On the other hand, norm $n_{4}$ forces a car to stop when it observes a car in front heading in the same direction of the reference car. This situation rarely leads to collisions, since the car in front rarely stops. As a consequence, its average necessity $\left(\bar{\mu}_{n e c}\right)$ has a low value $(0.33)$. We say then that this norm is preventive, since agents should comply with it in order to avoid collisions that rarely occur.

We have thus shown that IRON can successfully synthesise a compact normative system with high effectiveness.

5.2.2. Macro analysis. We now explore the limits of IRON by testing its synthesis capabilities under different norm infringement rates. Specifically, we analyse IRON's percentage of convergence for different norm infringement rates, ranging from 0.1 to 0.9 . We performed 100 simulations per norm infringement rate. Figure 7 shows averaged results for the effectiveness and necessity of the synthesised normative systems ${ }^{4}$

Moreover, the convergence degree series shows the number of runs that converged to a stable normative system out of the 100 runs. For low and medium norm infringement rates (up to 0.4 ), IRON's stability degree is 1, namely it successfully converged $100 \%$ of the times. Furthermore, it converged to normative systems with high effectiveness $(0.88)$ and necessity (0.71). Between medium and high norm infringement rates $(0.4$ and 0.7 ), the convergence decreases (due to oscillations in the normative systems),

${ }^{4}$ For the sake of clarity, we do not plot standard deviations. However, it is worth mentioning that the standard deviations for effectiveness and necessity for each norm infringement rate are within $[0.006,0.011]$ and $[0.080,0.0137]$ respectively. 


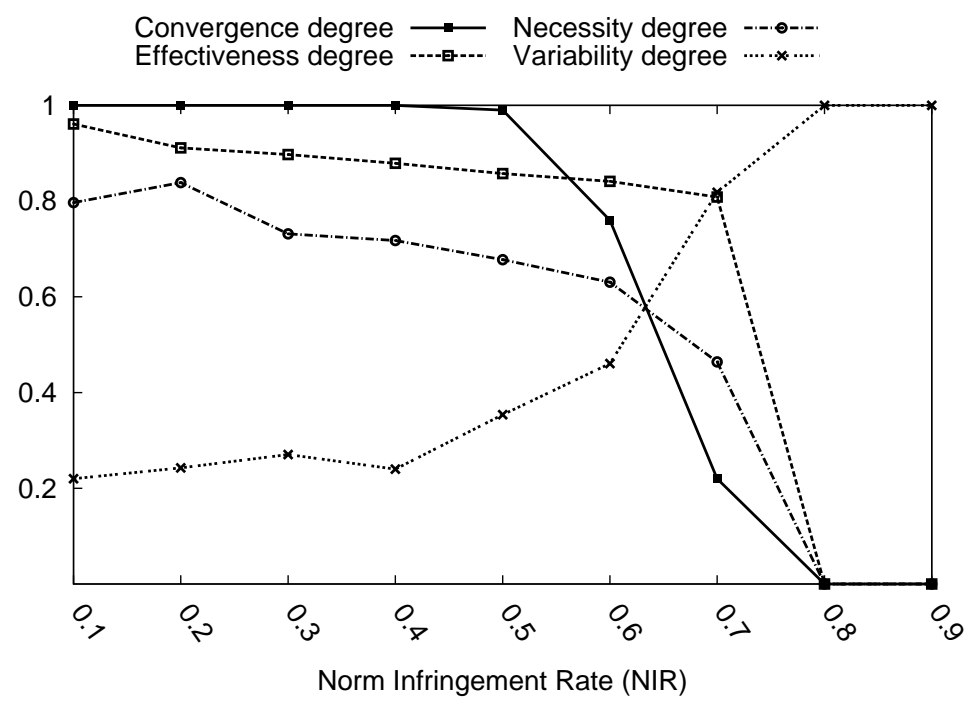

Fig. 7. Robustness analysis depending on norm infringements. The x-axis represents the different norm infringement rates, and the $y$-axis represents the different degrees that are given in the legend.

and it is for very high norm infringement rates (from 0.8 onwards, which means that agents decide to infringe norms 8 or more times out of 10 times) that IRON cannot find a normative system. Overall, IRON proved to be highly resilient to non-compliant behaviours during the synthesis process. IRON managed to successfully synthesise norms despite up to $40 \%$ norm infringement rate of agents.

Figure 7 also shows the variability of IRON's synthesis, namely whether it yields different normative systems. Below the 0.4 norm infringement rate, the variability remains near 0.2 (i.e., 100 executions converged to 20 different normative systems). Since preventive norms become unstable (activated and deactivated back and forth) with high norm infringement rates, IRON takes longer to synthesise stable norms. This leads to new, different normative systems, which IRON did not need to explore with lower norm infringement rates.

5.2.3. IRON versus BASE: Comparison of Stability and Compactness. Next we compare IRON with BASE, the mechanism for norm synthesis described in Section 3. Our experiments employ the publicly available version of BASE at [Morales et al. 2011b]. Our comparison employs the same traffic scenario described in Section 5.1 above, and the experimental settings for BASE described in [Morales et al. 2011c].

Stability degree. We first compare both norm synthesis approaches in terms of their stability. We performed 100 simulations per norm synthesis method. Our analysis is performed for very low (0.1) low (0.2), medium (0.3 to 0.6) and high (beyond $0.6)$ norm infringement rates. Figure 8 illustrates how normative systems change along a single, sample simulation: the switch frequency between different normative systems of BASE is much higher than IRON's, which stabilizes normative systems for longer periods of time until it converges. At the end of the simulation BASE explored 251 different normative systems and was not able to converge, while IRON explored 41 different normative systems, converging to a final normative system. Additionally, Figure 9 compares the stability degree of both norm synthesis methods over 100 simulations. For very low norm infringement rates $(0.1)$, both methods 


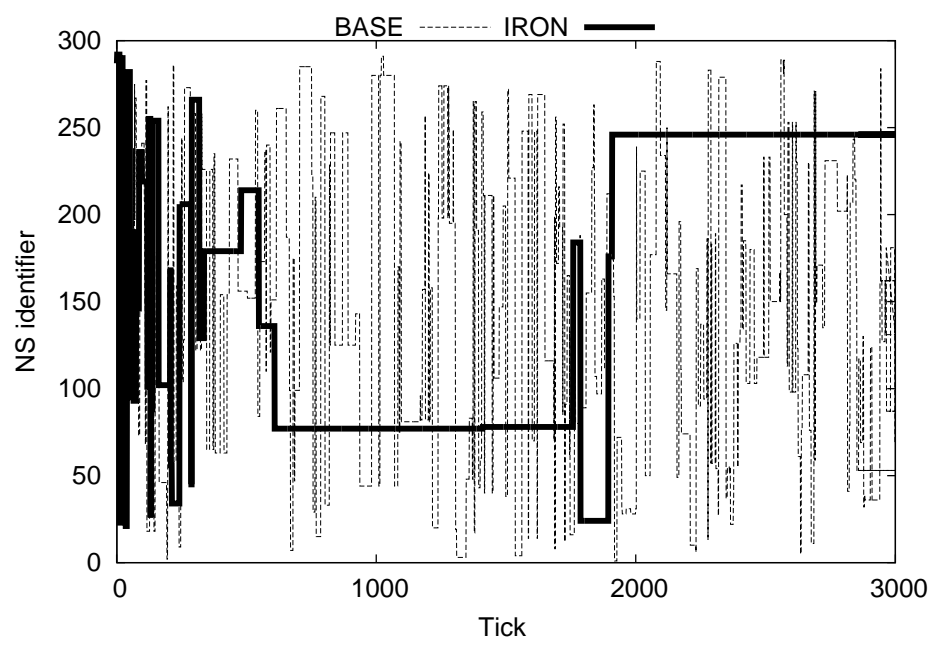

Fig. 8. Normative system changes along time.

successfully converge to a normative system that effectively coordinates the MAS. Nevertheless, beyond low norm infringement rates (0.2), BASE dramatically decreases its stability degree (i.e., it becomes more inefficient in synthesising a normative system that avoids collisions). In fact, it never manages to converge to a normative system beyond the 0.3 norm infringement rate, and collisions are never completely eradicated, hence leading to a 0 stability degree. As for IRON, it converges for low norm infringement rates and totally removes collisions $100 \%$ of the times. Still, for medium norm infringement rates (0.3 to 0.6$)$, its stability degree is between 0.9 and 1 , namely it converges between $90 \%$ and $100 \%$ of the simulations. The stability degree of IRON tends to decrease beyond high norm infringement rates (0.6), and it is for very high norm infringement rates $(0.8)$ that it fails to converge. Overall, IRON is much more stable than BASE, allowing to converge for much higher norm infringement rates.

Compactness savings. We now compare the compactness of the normative systems synthesised by IRON and BASE. Recall from Definition 5 that we measure the compactness of a normative system in terms of its overall number of norm predicates. Figure 10 illustrates the compactness savings achieved by IRON with respect to BASE: it saves compactness for all norm infringement rates, achieving its best results for medium norm infringement rates.

For low norm infringement rates, (up to $20 \%$ norm infringement rates) IRON manages to converge to normative systems that are between $30 \%$ and $40 \%$ more compact (have fewer norms) than those synthesised by BASE. As for medium norm infringement rates (0.3 to 0.6), IRON obtains its best savings in compactness (up to 70\%: from 36.2 down to 10.5 predicates on average). Here IRON benefits from its stability, whereas BASE is penalised by its instability. This is due to the fact that, when BASE does not converge, it typically outputs normative systems with a low compactness. Specifically, while IRON manages to converge between $90 \%$ and $100 \%$ of the time, BASE just converges $72 \%$ of the time for a norm infringement rate of 0.3 , while it never converges beyond 0.4 norm infringement rate. As a result, IRON synthesises normative systems that are up to $70 \%$ more compact than those synthesised by BASE. As for high norm infringement rates (beyond 0.6), IRON's savings in compactness tend to decrease since its percentage of convergence decreases as well. Overall, IRON synthesises normative 


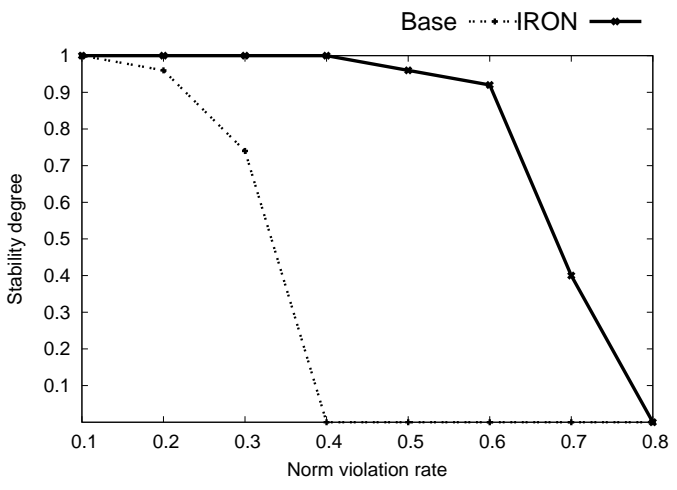
Fig. 9
BASE

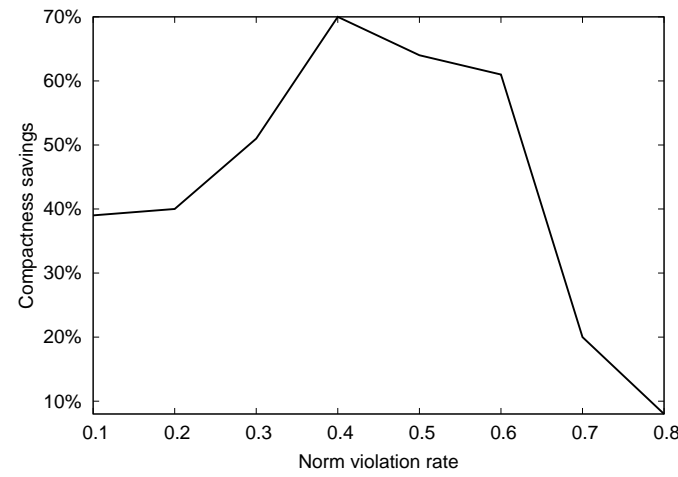

Fig. 10. Compactness savings of IRON with respect to BASE

systems that are much more compact than those synthesised by BASE, allowing agents to save computational resources when processing norms.

\section{CONCLUSIONS AND FUTURE WORK}

In this paper we have introduced IRON, an approach for the on-line synthesis of norms for multi-agent systems. IRON is a domain-independent architecture and computational model composed of three abstract elements: (i) a normative network to represent the norms generated during the synthesis process; (ii) a set of operators to apply changes to the normative network; and (iii) a strategy to perform norm synthesis. One can use IRON to perform norm synthesis on different domains by implementing relatively simple domain-dependent functions such as conflict detection and norm applicability.

We have performed an empirical evaluation of IRON on a simplified traffic junction scenario. In our scenario, cars are autonomous agents that drive through a junction to reach their destinations. The goal of IRON is to synthesise norms that prevent cars from colliding. Our empirical results show that IRON significantly outperforms BASE, the norm synthesis techniques presented in [Morales et al. 2011c]. On the one hand, we have shown that IRON's normative systems are up to $70 \%$ more compact than those generated by BASE. On the other hand, we have also shown that unlike BASE, IRON successfully synthesises normative systems despite high norm infringement rates. These improvements mainly derive from the fact that IRON takes more informed and fine-grained decisions than BASE. This comes at the cost of employing: (i) a generalisation function that requires complete evidence prior to perform norm generalisations; and (ii) a specialisation function that allows to perform a fine-grained backtracking of norm generalisations.

As future work, there are multiple opportunities for research. First, we plan to enrich our current scenario to include further traffic elements like pedestrians, cars driving at different speeds, or even traffic lights. Along this line, in [Morales et al. 2011a] we already performed an empirical evaluation that compared how BASE and traffic lights managed to regulate a traffic junction. We believe that it is worth pursuing this line of inquiry. Second, we plan to enhance IRON to synthesise norms while considering multiple goals at the same time, instead of a single goal as we do in this paper. For instance, norms could be synthesised also considering the traffic flow. That is, IRON could synthesise norms to (1) avoid car collisions; and (2) to avoid traffic jams. Notice that this setting is specially interesting to be studied, since both goals are contradictory. On 
the one hand, avoiding car collisions requires occasional car stops, which is prejudicial for the traffic flow. On the other hand, undoing a traffic jam requires cars to start moving, which can lead to occasional collisions. Third, and finally, we plan to investigate how to further improve compactness. This is motivated by our analysis of the normative systems synthesised by IRON. We have observed that several norms may apply to the very same state. In particular, we have found two new types of relationships that can be characterised as follows: (i) two norms apply to a state, but only one of them suffices to regulate the state (the other one is therefore superfluous); and (ii) two norms apply to a state, but they perform (in terms of effectiveness) worse together than separately. The first type of relationship is directly related to over-regulation: regulating a state with two norms, when only one of them is sufficient, implies to over-constrain the behaviour of agents. Thus, removing one of them may decrease over-regulation, hence increasing individual agents' freedom. The second type of relationship captures a "conflict" between norms: the two of them together in the normative system decrease the effectiveness of a normative system. From this discussion follows that capturing these two relationships will help us improve compactness, and eventually effectiveness.

\section{DISCUSSION}

In what follows we focus on providing pragmatic guidelines to help potential users decide when and how to employ IRON.

First, we analyse the requirements that a scenario, besides the traffic scenario described in this paper, must satisfy in order to apply IRON:

- The norm generation approach employed by IRON is based on CBR and thus, it assumes that similar problems (in our case, conflicts) have similar solutions (norms).

- MAS conflicts must be identifiable. Since IRON's norm synthesis is based on the detection of undesired states, it must be able to decide whether a state represents a conflict or not.

- Agents' actions must be observable and, in case they cause conflicts, these arise immediately. IRON currently assumes that whenever a conflict arises at time $t$, the cause of the conflict can be found at the action of an agent at previous time step $t-1$. Moreover, IRON also requires the agent causing the conflict to be involved in it at time $t$.

- The number of agents involved in a conflict must be limited. Before IRON generates a norm that successfully regulates a conflict, it may generate a different norm for each of the agents involved in it. This approach is only feasible if the number of agents is limited, and hence the number of possible norms to generate for a given conflict is limited as well.

- The number of norms that the grammar employed by IRON can generate must not be large. Recall that the computational complexity of IRON's synthesis is linear with the number of norms that a grammar can generate, but this number is exponential with the number of predicates and their arity.

In this work we have used a simplified traffic scenario to illustrate IRON. However, we argue that IRON can be employed in a vast variety scenarios whenever the conditions above hold. As an example, in [Morales et al. 2014] we show how to apply IRON to perform norm synthesis in an on-line community scenario. In that scenario, the users of the community upload and view contents, and they complain about those contents they find inappropriate for the community (e.g., spam, or porn contents). There, undesired states are those where a significant amount of users complain about a given content. We showed that IRON is capable of synthesising norms that prevent users from uploading conflicting contents. 
Secondly, we focus on how to tune the parameters employed by IRON. The guidelines that follow stem from a series of experiments that we conducted to understand the impact of the specialisation and generalisation thresholds on IRON's convergence. More precisely, we ran the empirical evaluation described in Section 5, but this time combining different specialisation thresholds (low, medium, and high values of $\alpha_{e f f}^{\text {spec }}, \alpha_{n e c}^{s p e c}$ ) with different generalisation thresholds (low, medium and high values of $\alpha_{e f f}^{\text {gen }}, \alpha_{n e c}^{g e n} \sqrt{5}$ Two main observations derive from our analysis:

- The value of IRON's specialisation (deactivation) thresholds directly impact on the type of norms that become part of synthesised normative systems. On the one hand, high specialisation thresholds lead IRON to include in a normative system those norms that are highly effective and necessary. Therefore, those norms that regulate situations that occasionally lead to conflicts (that is, that are slightly necessary) are never included. Thus, we should use high specialisation thresholds when we aim at very compact normative systems and we are ready to tolerate occasional conflicts. On the other hand, low specialisation thresholds lead IRON to include in a normative system any norm that avoids undesired states, even though the situation it regulates very sporadically leads to conflicts.

- The value of IRON's generalisation thresholds affect the compactness of synthesised normative systems. On the one hand, high generalisation thresholds mean that only those norms that are highly effective and necessary can be generalised. Since performing norm generalisations is costly, this setting is appropriate in scenarios where the cost of generalising norms is relevant. Thus, this setting guarantees that a norm is only generalised when it performs well. On the other hand, low generalisation thresholds allow IRON to generalise a norm even though its effectiveness and necessity values are low. This leads to increasing the compactness of the normative system.

\section{ACKNOWLEDGMENTS}

Work funded by AT (CONSOLIDER CSD2007-0022), EVE (TIN2009-14702-C02-01,TIN2009-14702-C0202), COR (TIN2012-38876-C02-01/02), MECER (201250E053) and the Generalitat of Catalunya (2009-SGR1434). Michael Wooldridge was supported by the ERC under Advanced Grant 291528 ("RACE").

\section{REFERENCES}

Agnar Aamodt and Enric Plaza. 1994. Case-Based Reasoning: Foundational Issues, Methodological Variations, and System Approaches. Artificial Intelligence Communications 7, 1 (1994), 39-59.

Cristina Bicchieri. 2006. The Grammar of Society: The Nature and Dynamics of Social Norms. Cambridge University Press.

Ken Binmore. 2005. Natural Justice. Oxford University Press.

Guido Boella, Leendert van der Torre, and Harko Verhagen. 2006. Introduction to normative multiagent systems. Computational \& Mathematical Organization Theory 12, 2-3 (2006), 71-79.

George Christelis and Michael Rovatsos. 2009. Automated Norm Synthesis in an Agent-based Planning Enviroment. In Proceedings of the 8th International Joint Conference on Autonomous Agents and Multiagent Systems (AAMAS'09). IFAAMAS, 161-168.

Frank Dignum. 1999. Autonomous agents with norms. Artificial Intelligence and Law 7, 1 (1999), 69-79.

David Fitoussi and Moshe Tennenholtz. 1998. Minimal social laws. In Proceedings of the National Conference on Artificial Intelligence (AAAI). John Wiley \& Sons LTD, 26-31.

Andrés García-Camino, Juan A Rodríguez-Aguilar, Carles Sierra, and Wamberto Vasconcelos. 2009. Constraint rule-based programming of norms for electronic institutions. Autonomous Agents and MultiAgent Systems 18, 1 (2009), 186-217.

\footnotetext{
${ }^{5}$ For the sake of keeping the paper length within reasonable limits, we did not incorporate this new set of experiments with different threshold values, but we do report on our main findings.
} 
Nathan Griffiths and Michael Luck. 2010. Norm Emergence in Tag-Based Cooperation. In 9th International Workshop on Coordination, Organization, Institutions and Norms in Multi-Agent Systems. (COIN'10). 80-87.

James E Kittock. 1993. Emergent conventions and the structure of multi-agent systems. L. Nadel and D. Stein, eds (1993).

John-Jules Ch. Meyer and Roel J. Wieringa. 1993. Deontic logic in computer science: normative system specification. John Wiley and Sons Ltd., Chichester, UK.

Javier Morales, Maite López-Sánchez, and Marc Esteva. 2011a. Evaluation of an automated mechanism for generating new regulations. In Proceedings of the 14th international conference on Advances in artificial intelligence: spanish association for artificial intelligence (CAEPIA'11). Springer-Verlag, Berlin, Heidelberg, 12-21.

Javier Morales, Maite López-Sanchez, and Marc Esteva. 2011b. Source code of Using experience to generate new regulations. http://www.iiia.csic.es/ jmorales/Downloads/Morales_IJCAI2011_SourceCode.tar. gz. (2011).

Javier Morales, Maite López-Sánchez, and Marc Esteva. 2011c. Using Experience to Generate New Regulations. In International Joint Conference in Artificial Intelligence (IJCAI'11). AAAI Press, USA, 307-312.

Javier Morales, Maite López-Sanchez, Juan A. Rodríguez-Aguilar, Michael Wooldridge, and Wamberto Vasconcelos. 2013. Automated Synthesis of Normative Systems. In Proceedings of the 12th International Conference on Autonomous Agents and Multiagent Systems (AAMAS'13). IFAAMAS.

Javier Morales, Iosu Mendizábal, David Sánchez-Pinsach, Maite López-Sánchez, and Juan A. RodríguezAguilar. 2014. Using IRON to Build Frictionless On-line Communities. AI Communications (To appear in the special issue of the Catalan Conference of Artificial Intelligence) (2014).

Stephen Muggleton and Luc De Raedt. 1994. Inductive Logic Programming: Theory and Methods. Journal of Logic Programming 19, 20 (1994), 629-679.

Shan-Hwei Nienhuys-Cheng and Ronald de Wolf. 1997. Foundations of Inductive Logic Programming. Springer-Verlag New York, Inc., Secaucus, NJ, USA.

Christopher K. Riesbeck and Roger C. Schank. 1989. Inside Case-Based Reasoning. Lawrence Erlbaum Associates, Hillsdale, NJ, US.

Norman Salazar, Juan A. Rodriguez-Aguilar, and Josep L. Arcos. 2010. Robust coordination in large convention spaces. AI Commun. 23, 4 (Dec. 2010), 357-372.

Onkur Sen and Sandip Sen. 2010. Effects of social network topology and options on norm emergence. In Proceedings of the 5th international conference on Coordination, organizations, institutions, and norms in agent systems (COIN'09). Springer-Verlag, Berlin, Heidelberg, 211-222.

Sandip Sen and Stéphane Airiau. 2007. Emergence of norms through social learning. In Proceedings of the 20th international joint conference on Artifical intelligence (IJCAI'07). Morgan Kaufmann Publishers Inc., San Francisco, CA, USA, 1507-1512.

Yoav Shoham and Kevin Leyton-Brown. 2009. Multiagent Systems: Algorithmic, Game-Theoretic, and Logical Foundations. Cambridge University Press, New York.

Yoav Shoham and Moshe Tennenholtz. 1995. On social laws for artificial agent societies: off-line design. Journal of Artificial Intelligence 73, 1-2 (February 1995), 231-252.

Munindar P. Singh, Matthew Arrott, Tina Balke, Amit K. Chopra, Rob Christiaanse, Stephen Cranefield, Frank Dignum, Davide Eynard, Emilia Farcas, Nicoletta Fornara, Fabien Gandon, Guido Governatori, Hoa Khanh Dam, Joris Hulstijn, Ingolf Krueger, Ho-Pun Lam, Michael Meisinger, Pablo Noriega, Bastin Tony Roy Savarimuthu, Kartik Tadanki, Harko Verhagen, and Serena Villata. 2013. The Uses of Norms. In Normative Multi-Agent Systems, Giulia Andrighetto, Guido Governatori, Pablo Noriega, and Leendert W. N. van der Torre (Eds.). Dagstuhl Follow-Ups, Vol. 4. Schloss Dagstuhl-Leibniz-Zentrum fuer Informatik, Dagstuhl, Germany, 191-229.

Richard S. Sutton and Andrew G. Barto. 1998. Introduction to Reinforcement Learning (1st ed.). MIT Press, Cambridge, MA, USA.

Daniel Villatoro, Jordi Sabater-Mir, and Sandip Sen. 2011. Social Instruments for Robust Convention Emergence.. In Proceedings of the 22nd International Joint Conference on Artificial Intelligence (IJCAI'11), Toby Walsh (Ed.). IJCAI/AAAI, 420-425.

Adam Walker and Michael Wooldridge. 1995. Understanding the Emergence of Conventions in Multi-Agent Systems.. In International Conference on Multiagent Systems (ICMAS'95). 384-389.

Chao Yu, Minjie Zhang, Fenghui Ren, and Xudong Luo. 2013. Emergence of Social Norms Through Collective Learning in Networked Agent Societies. In Proceedings of the 12th International Conference on Autonomous Agents and Multiagent Systems (AAMAS'13). IFAAMAS. 


\section{Online Appendix to: On-line Automated Synthesis of Compact Normative Systems}

JAVIER MORALES, Artificial Intelligence Research Institute (IIIA-CSIC), Spain

MAITE LÓPEZ-SÁNCHEZ, University of Barcelona, Barcelona, Spain

JUAN A. RODRIGUEZ-AGUILAR, Artificial Intelligence Research Institute (IIIA-CSIC), Spain

WAMBERTO VASCONCELOS, University of Aberdeen, United Kingdom

MICHAEL WOOLDRIDGE, University of Oxford, United Kingdom

The source code of the IRON norm synthesis mechanism is available on-line in http: //www.iiia.csic.es/ jmorales/Downloads/IRON.zip.

Finally, the traffic simulator employed in Section 5 to empirically evaluate IRON is available in http://www.iiia.csic.es/ jmorales/Downloads/TrafficSim.zip

(c) YYYY ACM 0000-0000/YYYY/01-ARTA $\$ 15.00$

DOI :http://dx.doi.org/10.1145/0000000.0000000 